\title{
Géolinguistique
}

21 | 2021

Varia

\section{À propos des dénominations de la salamandre d'Afrique du Nord : motivations, contact de langues et approche ethnobiologique}

About the Names of the North African Salamander: Motivations, Language

Contact and Ethnobiological Approach

\section{Massinissa Garaoun}

\section{OpenEdition}

Journals

Édition électronique

URL : https://journals.openedition.org/geolinguistique/5980

DOI : 10.4000/geolinguistique.5980

ISSN : 2650-8176

\section{Éditeur}

UGA Éditions/Université Grenoble Alpes

\section{Édition imprimée}

ISBN : 978-2-37747-303-8

ISSN : 0761-9081

\section{Référence électronique}

Massinissa Garaoun, «À propos des dénominations de la salamandre d'Afrique du Nord : motivations, contact de langues et approche ethnobiologique », Géolinguistique [En ligne], 21 | 2021, mis en ligne le 06 décembre 2021, consulté le 19 décembre 2021. URL : http://journals.openedition.org/ geolinguistique/5980; DOI : https://doi.org/10.4000/geolinguistique.5980

Ce document a été généré automatiquement le 19 décembre 2021.

Géolinguistique 


\title{
À propos des dénominations de la salamandre d'Afrique du Nord : motivations, contact de langues et approche ethnobiologique
}

\author{
About the Names of the North African Salamander: Motivations, Language \\ Contact and Ethnobiological Approach
}

Massinissa Garaoun

\section{Introduction'}

1 L'Afrique du Nord occidentale abrite un certain nombre de poches de populations relictuelles de salamandres, appartenant à un complexe de sous-espèces plus ou moins isolées géographiquement et génétiquement. Ce complexe, classé sous le nom scientifique de Salamandra algira, la salamandre algire, fut premièrement décrit en Algérie par Bedriaga (1883) à partir de la population du massif de l'Edough (massif littoral du Nord-Est algérien). Pensée premièrement comme endémique à l'Algérie, cet amphibien fut par la suite décrit dans des montagnes du nord du Maroc (Rif et Moyen Atlas septentrional), tandis que des recherches infructueuses au nord de la Tunisie semblent confirmer une répartition strictement maroco-algérienne (Bogaerts et al., 2013). Ce n'est qu'au début du $\mathrm{XxI}^{\mathrm{e}}$ siècle, que des chercheurs ont découvert l'existence d'une importante diversité intraspécifique de cet animal au nord du Maroc: avec pas moins de quatre nouvelles sous-espèces micro-endémiques, dont les répartitions sont parfois réduites à quelques vallons montagneux.

2 Jusqu'à présent, aucune étude ne s'est penchée sur la nomenclature de la salamandre en Afrique du Nord. Celle-ci présente pourtant plusieurs avantages : l'existence d'une unique espèce à répartition limitée à quelques massifs montagneux littoraux algéromarocains nous permet de travailler avec un nombre de points d'enquêtes relativement 
faible. Par ailleurs, la salamandre est un animal pour le moins spectaculaire, qui, malgré son rythme biologique l'amenant à passer inaperçu des hommes la plus grande partie du temps, est partout connue des populations rurales qui partagent son environnement. Enfin, contrairement à d'autres batraciens, et en particulier les anoures, les désignations de la salamandre ne sont pas envahies par les formes d'origines onomatopéiques imitant le coassement, lesquelles sont souvent difficiles à étudier d'un point de vue historique. À ces avantages, cette recherche zoonymique présente des intérêts auprès de différentes disciplines: l'étude des motivations lexicales additionnée à la documentation des légendes, pratiques et dictons concernant cet animal intéressent autant l'anthropologue, le linguiste, que le biologiste. La répartition de notre urodèle à cheval sur diverses aires berbérophones, dont plusieurs sont sous-documentées et menacées (Sanhaja de Sraïr, kabyle des Babors, de l'Atlas blidéen, etc.), apporte une motivation dans le cadre de la documentation de ces dernières. Enfin, la linguistique de contact se voit interrogée par le questionnement des stratégies prises dans les variétés d'arabes concernées pour dénommer une espèce animale endémique à l'Afrique du Nord. Notre article s'articulera, après un bref aperçu de l'écologie de cet animal, en une première partie linguistique s'intéressant à sa nomenclature, puis en une seconde partie portant plutôt sur les aspects ethnobiologiques comprenant de courts corpus concernant les dictons et les pratiques entourant notre animal, ainsi que leur comparaison à l'échelle circumméditerranéenne.

Figure 1. - Salamandra algira algira (Tamridjet, Béjäia : Algérie).

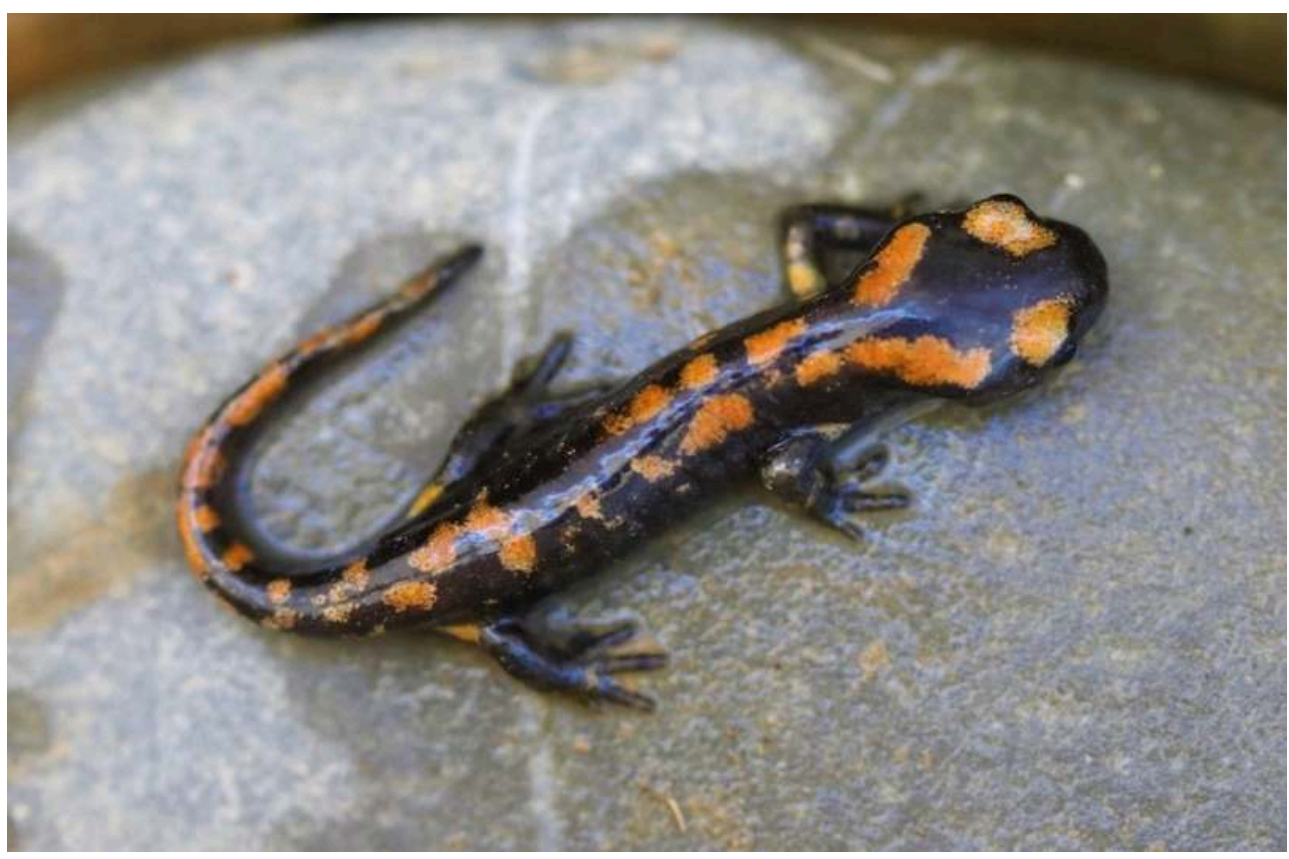

Photo : M. Garaoun.

\section{Présentation biologique}

Les salamandres sont des batraciens urodèles, une famille d'amphibiens se distinguant par la conservation d'une queue à l'âge adulte. La plupart des espèces de salamandres sont des amphibies strictes, c'est-à-dire que la croissance des animaux à partir de 
l'éclosion au stade larvaire (têtard) jusqu'au stade adulte et à la ponte n'est possible qu'au moyen de l'alternance d'une période de vie aquatique puis terrestre ${ }^{2}$. Ce sont des animaux carnivores, plus précisément insectivores. Ombrophiles voire nocturnes, les adultes chassent leurs proies à l'affût dans les habitats humides qu'ils affectionnent (humus, grottes, bois mort, etc.).

4 La salamandre algire adulte mesure entre 15 et $20 \mathrm{~cm}$ de long. Cet animal se caractérise par son aspect luisant et sa coloration originale, noire, tachetée de jaune, mais aussi de rouge chez certaines sous-espèces. Signalons enfin l'espérance de vie exceptionnelle des salamandres à l'échelle des urodèles, puisqu'elles atteindraient dans la nature une vingtaine d'années, jusqu'à trente ans en captivité.

Figure 2. - Fontaine du village d'Aït Aïssa (commune d'Aokas, Algérie), dans laquelle des salamandres se reproduisent chaque année.

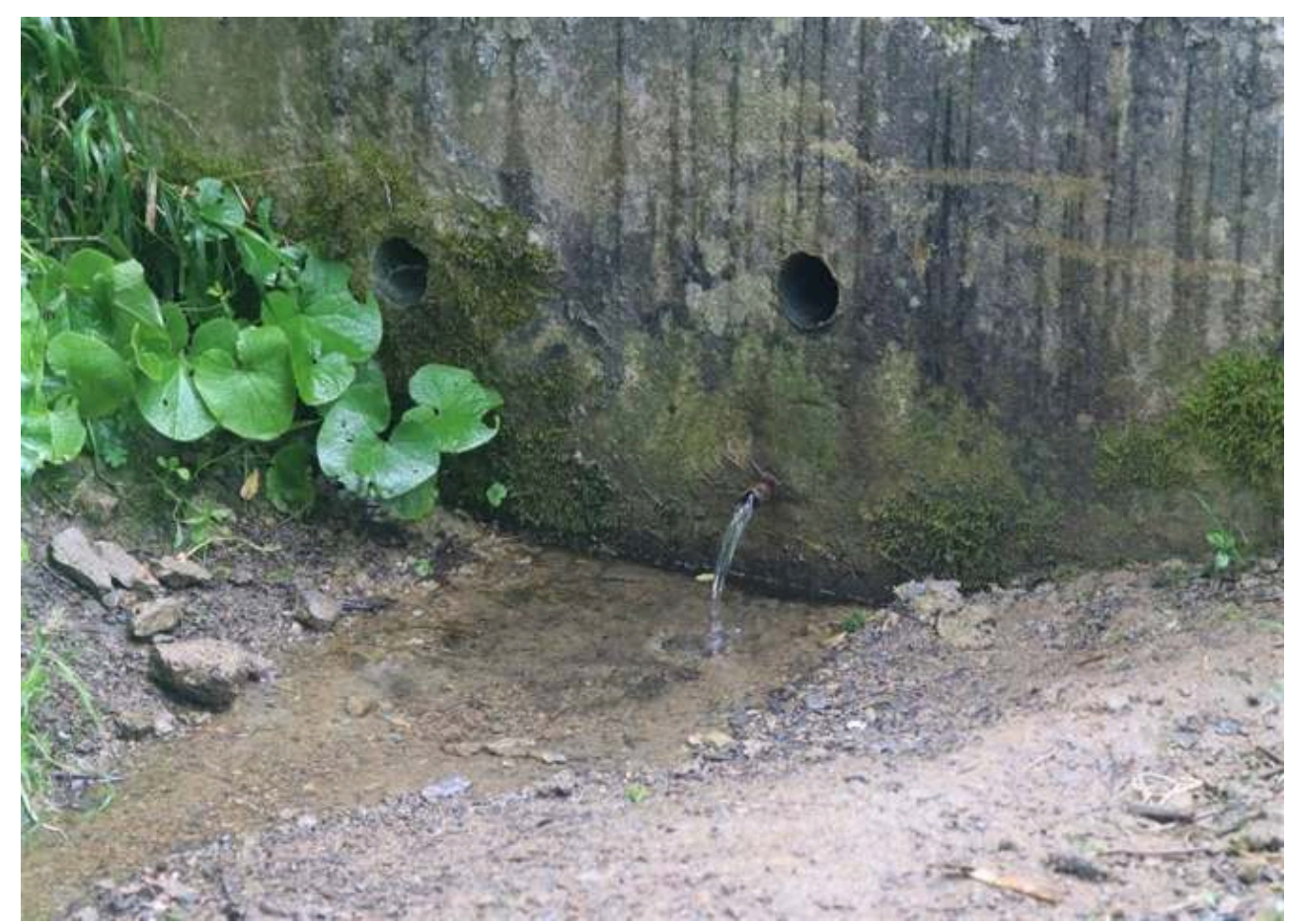

Photo : M. Garaoun. 
Figure 3. - Têtard de salamandre algire (Tamridjet, Béjäia : Algérie).

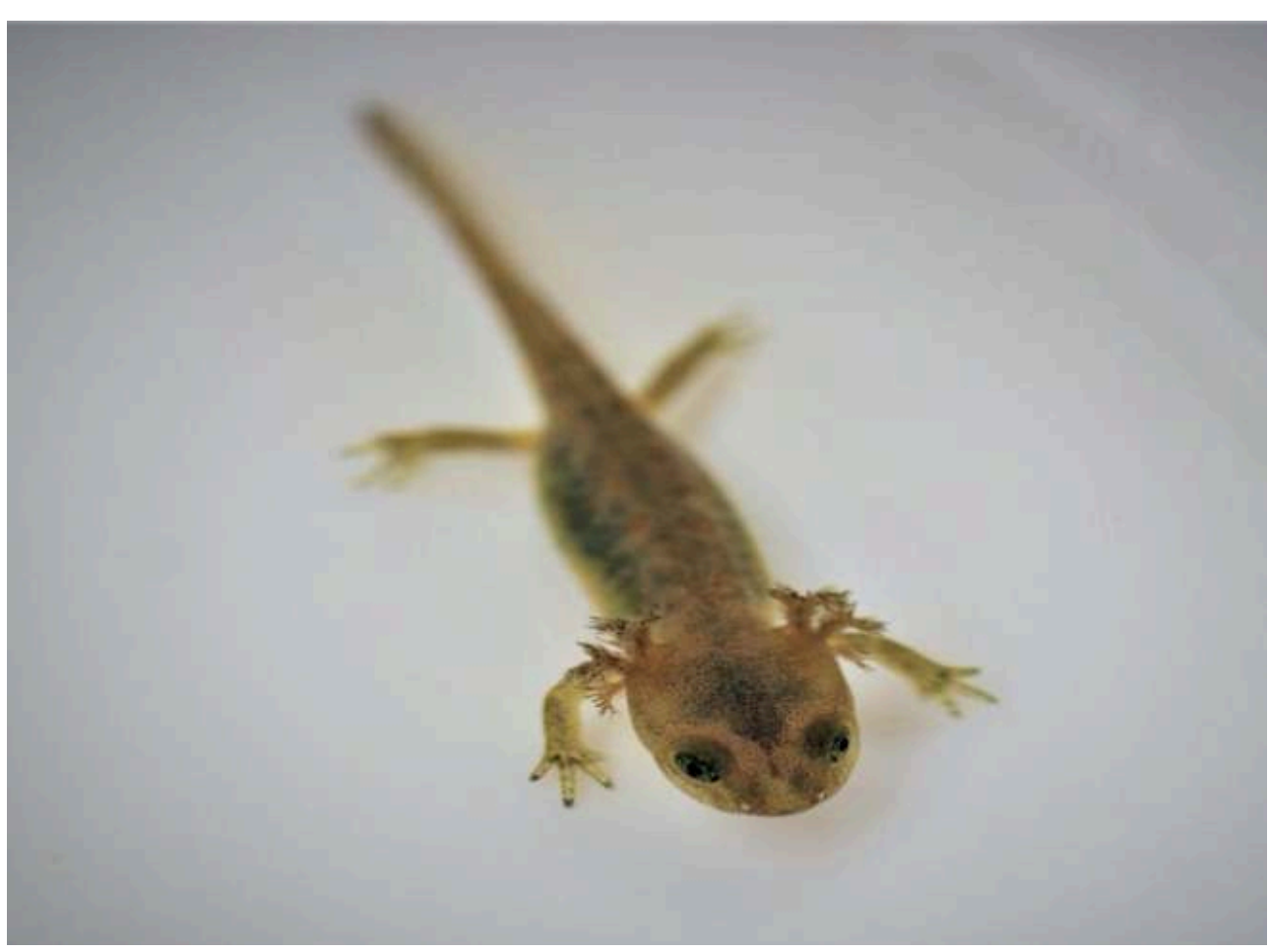

Photo : M. Garaoun.

Figure 4. - Juvénile de Salamandra algira algira (Aokas, Béjäia : Algérie).

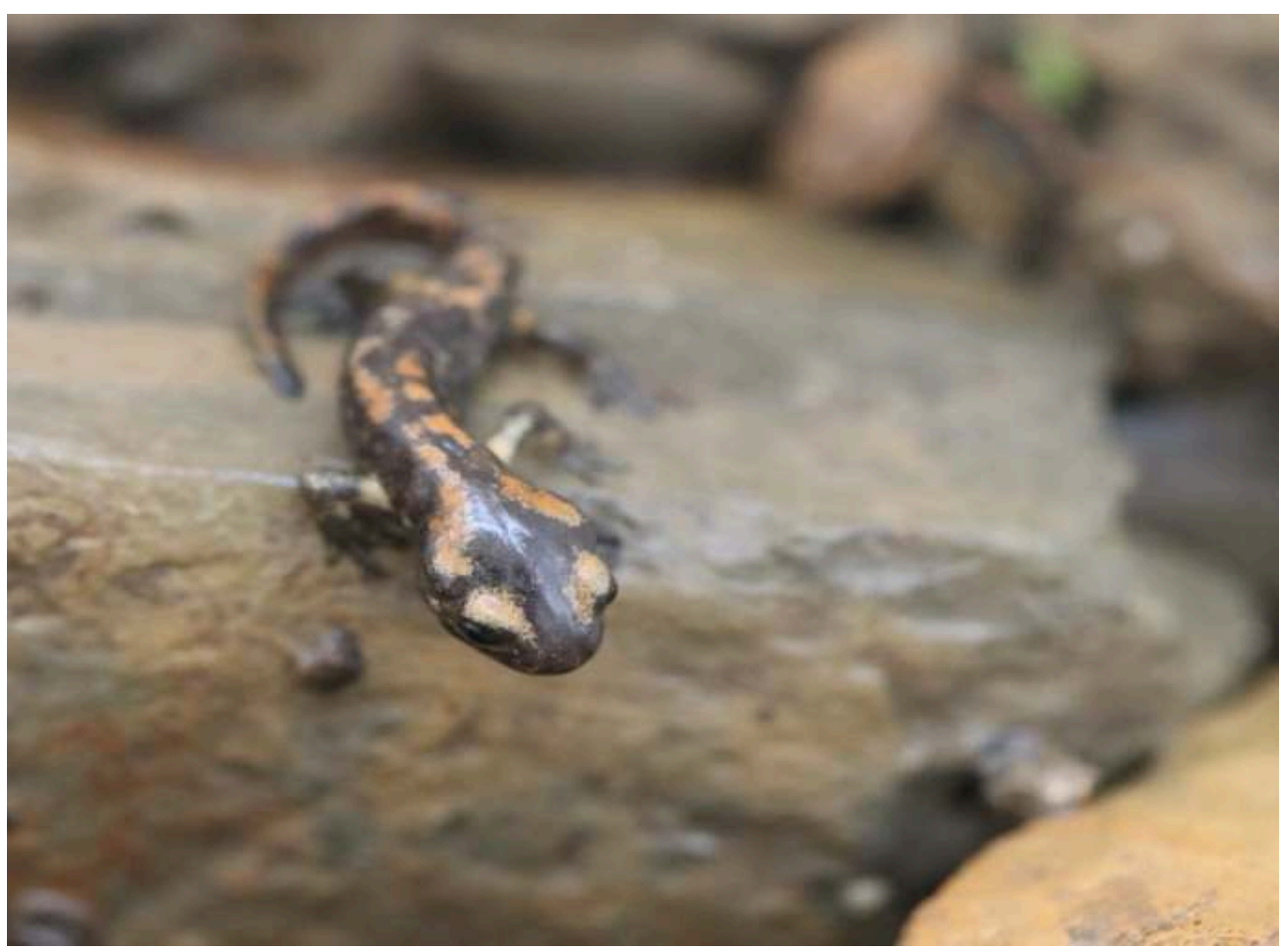

Photo : M. Garaoun. 


\subsection{Origine et diversité des salamandres en Afrique du Nord}

5 Le genre Salamandra comprend seulement sept espèces, lesquelles se rencontrent en Europe centrale, en Europe du sud, en Afrique du Nord ainsi qu'au Moyen-Orient. Salamandra algira est la seule parmi celles-ci à peupler l'Afrique du Nord, où elle n'est que faiblement répartie dans quelques massifs littoraux algériens et marocains. Selon Beukema et al. (2010), la conquête de ces régions par cet animal pourrait trouver son origine dans la crise de salinité messinienne ${ }^{3}$, laquelle a provoqué un assèchement de la méditerranée, et par conséquent la possibilité pour cet animal de conquérir le Maghreb occidental depuis la péninsule ibérique, sans doute via l'actuel détroit de Gibraltar. La salamandre algire présente une diversité intraspécifique importante générée par l'isolement de certaines populations dans les petits massifs du nord de l'Atlas où elles ont pu s'établir. Au Maroc, quatre sous-espèces ont été décrites durant la dernière décennie : S. algira tingitana au nord et à l'ouest de Tanger ainsi que dans la péninsule de la Ceuta ; S. algira splendens dans le Rif central, S. algira atlantica dans le nord et le centre du Moyen Atlas, et enfin S.algira spelaea dans l'unique massif des Aït Iznassen (Béni Snassen) du Rif oriental non loin de la frontière algérienne. En Algérie l'ensemble des formes de S. algira ont été associées au type (S. algira algira) décrit dans le massif de l'Edough par Lallemant en 1867. Il convient de dire que, contrairement aux populations marocaines, celles-ci s'observent tout le long du corridor écologique formé par l'ensemble des régions montagneuses dit des Kabylies, allant du massif blidéen à celui de l'Edough, et traversant le Djurdjura, les Babors et Collo. Des doutes subsistent néanmoins sur le statut de la population algérienne du massif des Traras (environs de Tlemcen).

\subsection{Habitat et répartition}

6 En Afrique du Nord, la salamandre colonise des habitats assez divers, entre les populations troglobies inféodées à des réseaux de grottes et de souterrain (S. spelea), les populations vivant au niveau de la mer du littoral méditerranéen (S. algira algira dans les Babors, S. algira tingitana dans le Rif occidental, etc.), ou encore celles franchement montagnardes observées à des altitudes parfois élevées (S.algira atlantica a été rencontrée entre 600 et 2455 mètres d'altitude dans le Moyen Atlas marocain). Les œufs et les larves de $S$. algira se développent dans l'eau fraîche des bassins d'eau stagnante en Kabylie où ils sont régulièrement observés dans des habitats d'origine anthropiques (fontaines, rigoles, bassins d'élevage de poissons). Les adultes sont difficiles à observer, le plus souvent leur rencontre se fait dans des conditions atmosphériques très humides (brouillard, pluie, orage, etc.). Dans certaines régions, nos informateurs nous ont confié qu'ils n'avaient jamais vu d'animaux vivants, mais uniquement écrasés par des véhicules sur les routes à la suite d'épisodes pluvieux. L'importance des populations de la salamandre algire varie également d'une station à une autre, dans le massif des Babors, région la plus humide d'Algérie, celle-ci est relativement commune et facile à observer en hiver. Une situation opposée à celle du massif des Traras, où l'animal est excessivement rare puisqu'il n'a plus été observé depuis sa découverte (Escoriza \& Ben Hassine, 2014). En Algérie et au Maroc, de nouvelles stations sont d'ailleurs fréquemment mises à jour, avec la découverte très récente (Hernandez \& Escoriza, 2019) de la sous-espèce S. algira atlantica dans le Moyen Atlas marocain, ou celle de 
populations troglobies dans la région littorale de Skikda en Algérie (Escoriza \& Ben Hassine, 2014).

\section{Les dénominations de la salamandre}

7 Nous décrirons ici les différentes appellations recueillies pour cet animal, et tenterons de les interroger sous le prisme de la sémantique lexicale, puis sous celui du contact de langue. Avant de regarder les données issues de notre enquête, nous présenterons quelques données nord-méditerranéenne, intéressantes pour la comparaison, aucune autre aire de répartition du genre Salamandra n'étant autant renseignée que celle-ci.

\subsection{Tour d'horizon circumméditerranéen}

8 Les dénominations de la salamandre autour de la méditerranée sont liées à la répartition des différentes espèces de cet animal: en Afrique du Nord, celles-ci se cantonnent à l'aire de répartition de S. algira (Maroc et Algérie); au Moyen-Orient, l'espèce S. infraimmaculata présente une aire de répartition très importante (Liban, Palestine, Syrie, Turquie, jusqu'en Irak et en Iran), et probablement de nombreuses appellations dans des langues très diverses. Enfin toute l'Europe centrale et méridionale jusqu'en Asie de l'Ouest est concernée par l'espèce Salamandra salamandra, relativement commune, dont les dénominations sont foisonnantes. Signalons enfin les salamandres noires alpines (S. lanzai et S. atra) ainsi que les deux micro-endémiques corse (S. corsica) et andalouse (S. longirostris).

Les données de l'Atlas linguistique roman (2009) nous ont permis d'accéder aux enquêtes linguistiques concernant le nom génétique des salamandres corses tachetées et noires. Nous ne regarderons pas les dénominations de cette dernière qui viennent généralement s'opposer à celle de la tachetée dans les régions où les deux espèces cohabitent. Nous donnerons également les quelques données recueillies pour le nom de l'espèce tachetée en arabe autrefois pratiqué en Andalousie. Parmi les langues d'Europe occidentale, signalons dès à présent l'origine du nom latin et scientifique salamandra, qui est un emprunt au grec $\sigma \alpha \lambda \alpha \mu \alpha ́ v \delta \rho \alpha$, lequel correspond lui-même à une composition conservée du substrat vasconique (Hickey, 2010: 890): *salam(a) « eau»+ and(e)ra «fille», littéralement "fille d'eau ». Cette étymologie est confirmée par le zoonyme basque relevé en synchronie ur-andra "demoiselle d'eau" (ibid.). Ces mots latins et grecs ont été intégrés par les langues savantes standardisées à diverses époques, et sont par conséquent actualisés par beaucoup de langues européennes (allemand salamander, hongrois szalamandra, bulgare САЛАМАНДЪР, provençal albreno, etc.), ainsi que dans quelques langues proches orientales (arabe classique السمندل, turque semenderi, etc.).

10 En dehors de cet étymon d'origine pré-indoeuropéenne, les cartes de l'Atlas linguistique roman concernant la salamandre (Nesi, 2001) divisent ses dénominations en 10 types sémantiques. Le premier, décrivant l'aspect de l'animal est à l'origine de la majorité des dénominations : morphologie de l'animal (aspect de la peau, présence de patte) :

- occitan biterrois (roman) blando «blonde» (Azaïs, 1877); charolais (roman) aubionde « blonde » (Rossi, 2006) ; bourguignon (roman) tè « tacheté » (ibid.), morvandiau (roman) tâ « tacheté » (ibid.). 
11 Le deuxième groupe sémantique le plus important est lié aux observations naturalistes des locuteurs, et lie la salamandre à ses habitats de prédilection (forêt, eau, etc.), ainsi qu'aux précipitations (pluie, orage, etc.) qui permettent souvent son observation (Nesi, 2001) :

- oill liz'ar d jo « lézard d'eau » (ibid.), kãnnl'ova « quand il pleut » (ibid. : 493), génois silv'estru « sylvestre » (ibid.), francoprovençal plāver'ula « pluie » (ibid.).

Cinq types sémantiques proposent dans le nom de la salamandre, des associations - peut-être distinctives de l'Europe méridionale - avec les nombreux mythes et pouvoirs qu'on lui attribue (grande venimosité, résistance au feu, etc., voir partie 4). Ces légendes sont généralement perçues négativement, de même que les appellations (Nesi, 2001):

- oil djabl « diable» (Nesi, 2001: 497), limousin (roman) essouble «souffleur, cracheur (de venin)» (Béronie \& Anne Vialle, 1824); normand (roman) mouron «souci, problème» (Termite, 2019) ; poitevin (roman) scorpion (Mauduyt, 1825) ; wallon (roman) rogne "gale " (Boutier, 2008); auvergnat (roman) enfleboeuf (Boujot, 2001); basque (vasconique) arrobio cognat de erensuge « dragon » (Orpustan, $2006: 14$ ).

D'autres appellations plus isolées sont motivées par des traits plus ou moins transparents. Plusieurs d'entre elles pourraient être liées à des croyances plus localisées ou perdues. Certaines insistent sur la laideur attribuée à cet animal, d'autres sont dérivées des dénominations d'autres animaux :

- saintongeais (roman) sereine « sirène, chanteuse » (Jônain, 1869: 41); berrichon (roman) sauret « lézard» (ibid.); corse catellu muntaninu (roman) « chiot de montagne» (Escoriza, $2007: 24$ ) ; champenois tet (roman) «qui tête» (confusion / superposition avec le têtard? (Tarbé, $1851: 136)$.

De l'arabe pratiqué ou anciennement pratiqué en Europe romane, nous n'avons que les traces de quelques dénominations relevées à travers les manuscrits d'arabe andalous, la salamandre étant absente de Sicile et de l'archipel maltais :

- Suhliyyah (Corriente, Pereira \& Vicente, 2017 : 621), donné comme une confusion avec le nom d'un reptile (ibid.) ; samandal (ibid. : 662), emprunté à l'arabe classique ; xemebráx (ibid. : 728), donné comme une composition < sāmmun abraṣ « venimeux et parsemé de taches » (ibid); távpat azugíc (ibid. : 828), dont le premier élément est un emprunt au roman talpa « taupe ", et le second est plus flou.

15 Signalons enfin le terme arrakl'an, un emprunt à l'arabe ( $<\varepsilon Q R B)$ signifiant à l'origine dans cette langue le "scorpion », est passé dans certaines variétés de castillan pour désigner la salamandre.

16 Cet aperçu des dénominations de l'animal dans des langues européennes a mis en exergue la diversité de celles-ci. Nous pensons qu'il serait tout à fait opportun de réaliser un travail parallèle pour nos données, et de classer les noms de cet animal selon leurs motifs sémantiques. Cet exercice pourrait mettre en évidence les principales correspondances sémantiques attribuées à cet animal à travers ses noms, et peut-être expliquer les conservatismes anciens observés.

\subsection{Les dénominations de la zone nord-africaine}

17 En Afrique du Nord, les noms de la salamandre sont relativement nombreux, bien que l'animal y soit plutôt rare et connu uniquement des populations rurales des régions où 
sa présence est attestée $e^{4 .}$ Nous avons tenté par divers moyens de recueillir ces dénominations partout où elles ont été signalées : pour cela, nous avons envoyés des questionnaires à une centaine de locuteurs via internet et interrogé directement ces derniers sur leur lieu de vie, dans les régions que nous avons le plus étudiées durant notre travail de terrain pour les besoins de notre thèse doctorale ${ }^{5}$ (massifs de la Kabylie des Babors, en Algérie). Dans ces questionnaires et durant ces entrevues, nous avons interrogé les locuteurs sur le nom de cet animal dans leurs parlers respectifs à partir de photographies de celui-ci. Afin de vérifier l'identification correcte de l'animal par le locuteur et afin d'éviter en particulier la confusion avec d'autres petits vertébrés, nous avons toujours demandé des informations sur l'écologie (réelle ou supposée) de la salamandre : « où vit la salamandre ? », « Quand l'aperçoit-on?», etc.

Ces questions ont par ailleurs souvent amené les locuteurs à partager avec nous les mythes et les croyances qui étaient associés à cet animal dans leurs communautés, éléments qui seront abordés dans la seconde partie de cet article. Par précaution, nous avons préféré ne pas mobiliser les sources lexicographiques existantes dans le cadre de cette enquête : les quelques formes glanées dans les dictionnaires et lexiques d'arabe maghrébin et de berbère semblent faire souvent l'objet d'erreurs d'identification ${ }^{6}$. De plus, les dictionnaires et lexiques un peu anciens de la zone berbère, ou encore ceux proposant typiquement des "standards " du berbère, donnent rarement l'origine précise dans laquelle ces termes ont été recensés et pourraient dans certains cas proposer des formes néologiques. Seules les formes trouvées dans deux dictionnaires ont été saisies dans ce corpus : celui des Aït Salah de l'atlas blidéen (El Arifi, 2016), ainsi que le dictionnaire du parler des At Manguellet du Djurdjura (Dallet, 1982). Dans le tableau suivant figurent les différentes variétés d'arabe maghrébin et de berbère parmi lesquels nous avons recherché la ou les dénominations de cet animal, en lien avec la sous-espèce concernée et donc son aire de répartition ${ }^{7}$.

\begin{tabular}{|l|l|l|}
\hline Sous-espèce & Variétés linguistiques & Pays \\
\hline S. a. algira & Ber./ar. des massifs des Kabylies, ar. des Traras? & Algérie \\
\hline S. a. tingitana & Ar. Jbala, ber./ar. des Ghomaras & Maroc \\
\hline S. a. splendens & Ber./ar. Sanhadji de Sraïr, rifain oriental & Maroc \\
\hline S. a. spelae & Rifain occidental, zénète de l'oriental marocain & Maroc \\
\hline S. a. atlantica & Ber. du Moyen Atlas, surtout du Moyen Atlas oriental & Maroc \\
\hline
\end{tabular}

19 En comparaison avec la répartition connue de la salamandre algire, nous étions étonnés de rencontrer une certaine difficulté à recueillir ses dénominations dans deux régions : le Rif occidental (pays Jbala) et le Moyen Atlas marocain. Dans le Moyen Atlas, cette difficulté pourrait être liée au mode de vie souvent entièrement troglodyte de l'animal (Hernandez \& Escoriza, 2009). Dans le pays Jbala, pour lequel nous disposons d'un faible nombre d'informateurs, nous pensons qu'il serait nécessaire de réaliser des enquêtes de terrains plus fournies. Inversement, nous avons été agréablement surpris de découvrir des appellations pour cet animal dans deux régions où celui-ci n'a pas été encore été observé par les biologistes : l'îlot septentrional chaouïphone des Amouchas (nord des 
hauts plateaux sétifien) et celui des At Menaceur (Dahra orientale). L'ensemble des 100 appellations recueillies sont présentées dans le tableau figurant ci-dessous ${ }^{8}$ :

\begin{tabular}{|c|c|c|}
\hline $\begin{array}{l}\text { Commune (+ village / } \\
\text { confédération) }\end{array}$ & Aire variétale & Dénomination(s) \\
\hline Tanger & Arabe jbala & $\varepsilon \bar{a} r \partial q \partial \underline{d}-\underline{d} b \bar{a} b$ \\
\hline Chefchaouen & Arabe jbala & $\varepsilon \bar{a} r u \bar{s} s$ ə̌̌ššta \\
\hline (Ġmāra) & Arabe jbala (Ghomaras) & bərqāța/bərțāța d əs-sma \\
\hline (Mezyaz) & Arabe jbala (Kétamas) & nəjmət əs-sma \\
\hline (Wahciyet) & Arabe jbala (Kétamas) & mzīwwqa \\
\hline (Tamsawt) & Arabe jbala (Kétamas) & jəmrət $\partial s-s m a$ \\
\hline (Bni Eisi) & Senhadji de Sraïr (Kétamas) & idiri $\sim$ ider \\
\hline (Bni ḥmed) & Senhadji de Sraïr (Kétamas) & axxuy n ccta \\
\hline (Ssahel) & Senhadji de Sraïr (Kétamas) & addar n genna \\
\hline (Bni Ḥmed) & Senhadji de Sraïr & adar $/$ itri $n$ tawwet \\
\hline (Ay Bunșar) & Senhadji de Sraïr & adar $n \underline{t}$ tagut \\
\hline (Ay Seddat) & Senhadji de Sraïr & lmarța $\sim$ lbarța adar n ccerqi \\
\hline (Zerqet) & Senhadji de Sraïr & adar $/$ baka $n$ taggut \\
\hline (Igzennayen) & Rifain occidental & da n tayut \\
\hline Ajdir (Ayt Waryayer) & Rifain occidental & aḍaḍ $n \underline{t a g g u}(y) \underline{t}$ \\
\hline (Ayt Iznasen) & Rifain oriental & azermița \\
\hline El Menzel (Bni yazġa) & Arabe des Bni yazgha & $\varepsilon \bar{a} b d$ əs-sma \\
\hline Tazekka (Ayt Warayen) & Tamazight du Moyen Atlas oriental & amejnun $n$ tayut \\
\hline Zkara (Ayt Zekri) & $\begin{array}{l}\text { Zénète de l'oriental } \\
\text { marocain }\end{array}$ & mjun n tayyut \\
\hline Menaceur (At Mnașer) & Zénète de la Dahra & filțaw \\
\hline Béni-Salah (Ayt Șaleḥ) & Tachelhit de l'Atlas blidéen & afiltu \\
\hline $\begin{array}{l}\text { Chabet el Ameur } \\
\text { (At Xalfun) }\end{array}$ & Kabyle extrême-occidental & awlul igenni \\
\hline Chabet el Ameur (At Eli) & Kabyle extrême-occidental & Awlul \\
\hline
\end{tabular}




\begin{tabular}{|c|c|c|}
\hline Timezrit (IYemrasen) & Kabyle extrême-occidental & Awlul \\
\hline $\begin{array}{l}\text { Sidi Ali Bounab } \\
\text { (Iflisen umellil) }\end{array}$ & Kabyle extrême-occidental & țașabant bbwaman \\
\hline Béni Amran (It Eamran) & Kabyle extrême-occidental & $\begin{array}{l}\text { abususihan tislit } \\
\text { ggemqwerqwar }\end{array}$ \\
\hline Draâ El Mizan & Kabyle extrême-occidental & tisdest bbwaman \\
\hline Tirmitin (Izerruden) & Kabyle extrême-occidental & tifixert \\
\hline Maatkas (Imactuqen) & Kabyle occidental & tifiyert \\
\hline Aïn Zaouïa (Bumahni) & Kabyle occidental & taqjunt igenni \\
\hline Makouda (At Wagennun) & Kabyle occidental & taqjunt uzemmur \\
\hline Souk El Thenine & Kabyle occidental & tidest bbwaman \\
\hline Aït Yenni (At Yenni) & Kabyle occidental & tigeltest bbwaman \\
\hline $\begin{array}{l}\text { Béni Zmenzer } \\
\text { (At Zmenzer) }\end{array}$ & Kabyle occidental & tigdest $\sim \underline{t i s d e s}^{9}$ \\
\hline Ouadhia (Iwaḍiyen) & Kabyle occidental & țagudreȚȚ ppwaman \\
\hline Larbaâ Nath Irathen (At Iraten) & Kabyle occidental & tigdezt bbwaman \\
\hline Ouacif (At Wasif) & Kabyle occidental & tayydest/tifidest bbwaman \\
\hline Aït Toudert (Tachechat) & Kabyle occidental & tigfest bbwaman \\
\hline Iboudraren (Ibudraren) & Kabyle occidental & tigzelt bbwaman \\
\hline Iferhounène (At Itsuray) & Kabyle occidental & taydest bbwaman \\
\hline Freha (At Ǧennad) & Kabyle occidental & tifidest bbwaman \\
\hline $\begin{array}{l}\text { Aïn El Hammam } \\
\text { (At mangellet) }\end{array}$ & Kabyle occidental & $\begin{array}{l}\text { taydest/tagdest/tigdest } \\
\text { bbwaman }\end{array}$ \\
\hline $\begin{array}{l}\text { Kabyle occidental } \\
\text { (Agwni } \mathrm{n} \text { testant) }\end{array}$ & Kabyle occidental & tidazt bbwaman \\
\hline Azazga (IEezzugen) & Kabyle occidental & tabureqmant \\
\hline El Asnam (At Yecla) & Kabyle occidental & tagrest bbwwaman \\
\hline Saharidj (Imesdurar) & Kabyle occidental & tizellit bbwaman \\
\hline Saharidj (Imcedallen) & Kabyle occidental & tigdest bbwwaman \\
\hline
\end{tabular}




\begin{tabular}{|c|c|c|}
\hline M'chedallah (Imcedallen) & Kabyle occidental & tiditit bbwwaman \\
\hline Takerboust (Takerbust) & Kabyle occidental & tisemsemt ggwaman \\
\hline Beni Mellikeche (At Mlikec) & Kabyle oriental & tayde \\
\hline Akbou (At Eebbas) & Kabyle oriental & tilest ggwaman \\
\hline El-Maïn (Ayt Yacla) & Kabyle oriental & taylest ggwaman \\
\hline Tazrout (At Wizgan) & Kabyle oriental & timreqqemt \\
\hline Sidi Aïch (At Weylis) & Kabyle oriental & tadist ggwaman \\
\hline Ouzellaguen (Uzellagen) & Kabyle oriental & tadayes ggwaman \\
\hline Tifra (Ikeǧğan) & Kabyle oriental & tislit ggwanzar \\
\hline Tifra (At Manșur) & Kabyle oriental & tisliț igenni \\
\hline Béni Yala (It Iعala) & Kabyle oriental & tigdest \\
\hline Béni Ouertilan (It Wertilan) & Kabyle oriental & abussihan \\
\hline El-Kseur (Ibarisen) & Kabyle oriental & țilețŢ iyenni \\
\hline Toudja (Tarḍam) & Kabyle oriental & țilseţ igenni \\
\hline Timezrit (At Yemmel) & Kabyle oriental & tislit wwaman \\
\hline Kendira (Ihbachen) ${ }^{10}$ & Kabyle oriental & aboușiḥ \\
\hline Tichy (It Mellul) & Kabyle oriental & țtir igenni $i^{11}$ \\
\hline Tizi N’Berber (It Bucisi) & Tasahlit occidental & lehlal n tyerza \\
\hline Aokas (Ayt Mhend) & Tasahlit occidental & țtir igenwan \\
\hline Tamridjet (Ayt Buysef) & Tasahlit occidental & tațarett $n$ igenni \\
\hline Kherrata (Ijermunen) & Tasahlit septentrional & tagțit igenni \\
\hline Aït Smaïl (Ayt Smaعel) & Tasahlit septentrional & tațarett $n$ igenni \\
\hline Tamridjet (Ayt Leelam) & Tasahlit oriental & țergenni \\
\hline Melbou (Ayt Segwal) & Tasahlit oriental & itțergenni atțergenni(t) \\
\hline Bouqalâ (Adewwaṛ Qelદun) & Chaoui des Amouchas & țamaqqast $n$ waman \\
\hline $\begin{array}{l}\text { Ziama-Mansouriah } \\
\text { (Bni Eīsa) }\end{array}$ & Arabe jijélien occidental & Abūryūn \\
\hline
\end{tabular}




\begin{tabular}{|c|c|c|}
\hline $\begin{array}{l}\text { Erraguène Souci } \\
\text { (Bni Mərmi) }\end{array}$ & Arabe jijélien occidental & țirūgənnān \\
\hline El Aouana (əl દāwāna) & Arabe jijélien occidental & țərgənni țirūgənna \\
\hline El Aouana (Bni Səkfāl) & Arabe jijélien occidental & țirūgənnār \\
\hline Jijel-ville & Arabe jijélien central & $a b \bar{a} y q \bar{a} \varepsilon \sim a b \bar{r} r y \bar{u} n$ \\
\hline Béni Habibi (Bni Ḥbībi) & Arabe jijélien oriental & abūryūn $\sim$ būryūna \\
\hline Boudriaa Béni Yadjis (Bni Yāğīis) & Arabe jijélien méridional & $b \bar{a} y q \bar{a} \varepsilon$ \\
\hline Djimla (Bni Fūgàal) & Arabe jijélien méridional & $b \bar{a} y q \bar{a} \varepsilon$ \\
\hline Oued Athmania & Arabe jijélien méridional & qerțāt əl-jənna \\
\hline Seraïdi & Arabe de l'Edough & wəld ər-rcād (mrāngət) \\
\hline
\end{tabular}

\subsubsection{Analyse dialectologique}

Les traits sémantiques relevés relatifs aux noms de la salamandre sont divers et variés, nous les avons classés en quatre groupes selon leurs degrés d'occurrences ainsi que leurs répartitions parmi les différentes aires dialectales arabes et berbères :

- Motifs isolés (observé(s) dans une seule aire) ;

- Motifs isolés redondants (rares mais observés dans plusieurs aires);

- Motif en bloc isolé (trait largement attesté dans une même aire) ;

- Motif en bloc redondant (trait largement attesté dans plusieurs aires).

21 La majorité des dénominations recueillies correspond au premier type, motifs rares et isolés, observés dans une seule aire linguistique. Les appellations rencontrées en bloc ne sont quant à elles représentées que par deux cas correspondants chacun à l'un des deux types décrit ci-dessus. Enfin, les appellations isolées mais redondantes bien que peu nombreuses sont d'un très grand intérêt : puisque celles-ci pourraient renvoyer à des formes dispersées par l'action des migrations et du contact entre les populations. Les différents motifs relevés sont représentés dans la carte suivante : 
Figure 5. - Carte des motifs sémantiques recueillis.

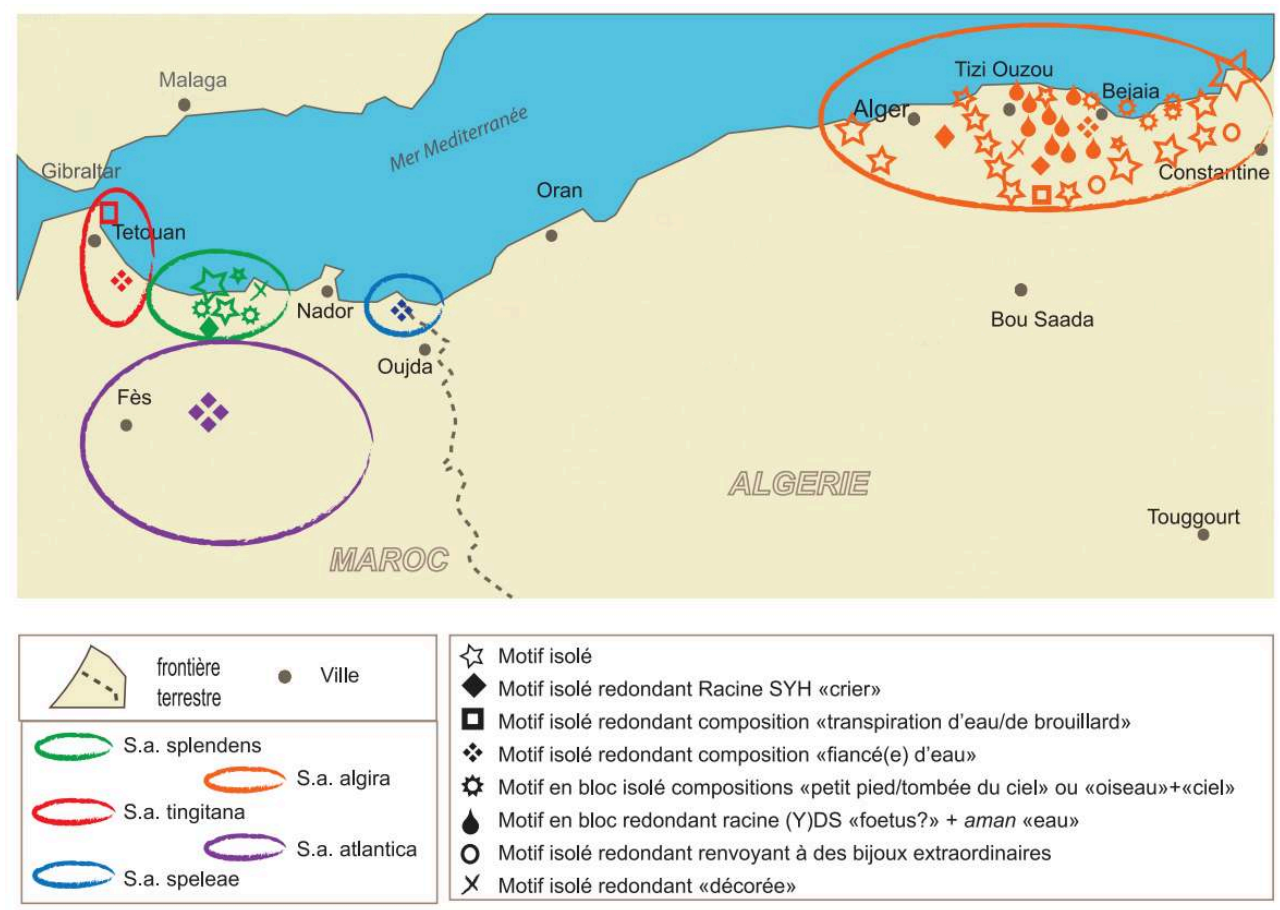

Photo : M. Garaoun

\subsubsection{Le bloc isolé $(F / Y) D S$ « fœtus ? » + aman « eau »}

Ce bloc de dénominations est largement réparti en Kabylie (Algérie) à travers le massif du Djurdjura et la vallée de la Soummam. Celui-ci présente des formes relevant d'une suite (F/Y)DS, le plus souvent observée en composition du nom aman « eau ».

La racine DS est attestée à l'échelle pan-berbère, où celle-ci est le plus souvent attachée au sème de ventre: Chleuh ad(d)is «ventre, Moyen Atlas adis "ventre, grossesse, fœtus ", tadist « bouche à nourrir, personne à charge, diarrhée, bas-ventre, entrailles ", tadist tadiss tédiss tadess " ventre", Neffoussa tiddist taddist ddiset ddist « ventre» (Naït-Zerrad, 1999 : 401). Les dérivés kabyles les plus fréquemment admis de cette racine sont tadist "grossesse, fœetus " (ibid.) et idis " côté, partie latérale d'une chose » (Kireche, $2010: 69$ ) qui est attesté en plusieurs points du Djudjura à travers la composition tadist $n$ waman dans la dénomination. Les locuteurs employant cette composition interprètent sa signification d'origine comme correspondant à «f fotus d'eau ».

D'après une proposition faite par Amazigh Bedar ${ }^{12}$, la suite YDS pourrait à son tour découler d'une suite FDS dont la première radicale se serait semi-vocalisée ${ }^{13}$ avant de chuter. Cette hypothèse est corroborée par la forme tifidast, récoltées en deux points de la Kabylie du Djurdjura. La suite FDS est d'ailleurs observée comme cognat de DS 《 ventre » dans le chleuh afddis « ventre (péjoratif) " (Naït-Zerrad, $2002: 529)$.

D'après ces différentes hypothèses, les formes relevées pourraient correspondre d'un point de vue diachronique au développement suivant: FDS (ex. tifidast) > YDS (ex. taydest) > DS (ex.tadist). À côté de ces formes, il existe des variantes relevant de dérivations phonétiques bien connues :

- consonnantification de [j] en [g], tendance caractéristique du kabyle occidental (ex. tigdest) ; 
- voisement de [s] en [z], phénomène universel fréquemment observé dans les parlers de la région ${ }^{15}$ (ex. tidazt bbwaman).

D'autres dénominations le plus souvent isolée, trouvent leur origine dans la corruption de la suite (F/Y)DS par chute ou l'interversion consonantique :

- tadayes wwaman : *YDS > DYS (métathèse) ;

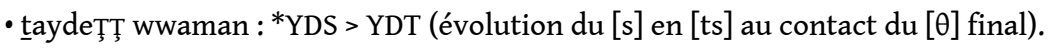

Nous avons noté un cas de réinterprétation de YDS par une autre racine :

- tadlest waman « diss d'eau » : *FDS > DLS < adles « diss ${ }^{16}$.

Le bloc de dénominations kabyles de la salamandre en F/YDS + « eau » est remarquable $\mathrm{du}$ fait de sa densité et de sa répartition circonscrite au cœur de la Kabylie berbérophone. L'existence de formes fortement corrompues phonétiquement voir réinterprétées dans d'autres racines pourrait être un gage de sa relative ancienneté.

\subsubsection{Le bloc redondant $\mathrm{DR}$ « pied/tombé » + GNW « ciel »}

29 Le second bloc de dénomination relevé est quant à lui redondant. Celui-ci correspond à des dénominations recueillies en Algérie, dans le berbère et l'arabe de la Kabylie des Babors, ainsi qu'au Maroc dans le Rif central, à travers l'aire également bilingue des Sanhadjas de Sraïr et des Kétamas. Un intérêt tout particulier de ce second bloc est lié au caractère bilingue des aires concernées. En effet, dans la Kabylie des Babors sont pratiqués à la fois différents parlers berbères (kabyles, chaouis et tasahlit), ainsi qu'un arabe de type préhilalien villageois connu pour constituer l'une des variétés d'arabe maghrébin ayant le plus largement emprunté à son substrat berbère ${ }^{17}$. Même situation dans le Rif central ou plusieurs variétés de berbère (Sanhadja de Sraïr, Kétama et rifain occidental) avoisinent l'arabe préhilalien dit jbala. Dans ce bloc, l'ensemble des dénominations correspondent à deux compositions liées entre elles par une histoire complexe de réinterprétation due au contact arabe-berbère : "pied (*tombant ?) »+ «ciel» et «oiseau » + «ciel ». En effet, la dénomination de la salamandre la plus fréquente en berbère tasahlit correspond au complexe entièrement de fonds berbère tațarett « diminutif de pied: petite patte, pied d'enfant, peton», n génitif; et igenni «ciel ».

Cette composition est motivée selon nous d'une part par la légende selon laquelle la salamandre «tombe du ciel avec la pluie $»^{18}$ (voir sous-partie 4.2), ce qui explique non seulement le second élément "ciel », et aussi par le choix du nom "pied» lequel appartient à une racine $\mathrm{D} / \mathrm{DR}$ «descendre, tomber, jambe, pied». D'après Chaker (1996 : 238) la racine DQR observée dans le nom $\operatorname{atar}^{19}$ « jambe, pied » correspond historiquement à une variante expressive de la racine $\mathrm{DR}$, laquelle est attestée à l'échelle pan-berbère pour les sèmes de «descendre » et de "presser » (Haddadou, 2007 : 40). Dans certaines variétés de berbère, les deux racines à emphase / sans emphase cohabitent, et sont à l'origine d'unités lexicales dont le lien sémantique est équivoque : kabyle ader «descendre » der «tomber », adar " pied, jambe » (Chaker, 1996: 238). Pour ces raisons, nous pensons que dans les dénominations de la salamandre recueillies dans les Babors, le choix de la suite DR «pied, patte» correspond à une réanalyse du sème issu de la même racine correspondant à l'action de «descendre»: ater (nom verbal atray). On dit en effet dans l'ensemble des communautés des Babors que la salamandre descend du ciel : Aït Bouysef tatarett igenni tettatar-edd zeg igenni (v. partie sur les pratiques, mythes et légendes) ${ }^{20}$. Cette analyse 
est renforcée par les dénominations recueillies à la frontière entre le berbère tasahlit et l'arabe jijélien :

- Tasahlit : Aït Segoual ițtergenni atțergenni( $(t)$, Aït Laâlam țergenni ;

- Arabe jijélien : El Aouana țərgənni, Bni Mermi țirūgənnān.

Ces formes contiennent à nouveau la racine $\mathrm{D} / \mathrm{DR}$, soudée à la racine GN « ciel ». Nous

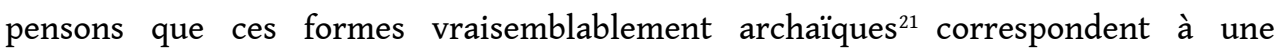
composition interprétable dans «chute, descente du ciel». Concernant la forme des Aït Segoual, signalons que l'élément /ț/ suffixé et facultatif pourrait correspondre à une contamination par la racine berbère DW : touareg ad "plier», enned "tourner, changer de direction", amenennad "homme tortueux", kabyle enned "entourer, s'enrouler », hennuned «tourner, trainer» (Haddadou, 2007: 50). La composition au moyen de cette racine est par ailleurs observable dans la région dans les formes azermammuț (tasahlit) azrəmțīwən (arabe jijélien) «lombric » toutes deux dérivées d'une racine initiale S/ZRM (kabyle aslem « poisson », azrem «serpent»), ainsi que la dénomination de la salamandre observée chez les Aït Iznassen (azermita). Ajoutons ici que des compositions plus ou moins figées composées du berbère ațar " pied, patte " sont également connues dans un phytonyme renvoyant à diverses espèces de plantes provenant du figement du berbère atar " pied» et de aylal «oiseau » (Tilmatine, $2005: 9)$ :

- Région de Fès (arabe) ațilan ațrilal (El Ghassânî, 1985 : 256);

- Tasahlit (Aït Segoual) traylal22.

32 À partir de la composition caractéristique des Babors "pied/ tombé du ciel ", un certain nombre de parlers berbères et arabes de la région ont généré, à partir d'un phénomène subtil de réinterprétation à partir de l'arabe, un second composé « oiseau / passereau du ciel » (ex. tasahlit tagtiti igenni « passereau du ciel»). Nous reviendrons sur les formes nées de ce phénomène dans la sous-partie 3.2.6.

Dans le Rif central, les compositions renvoyant aux sèmes de "pied du ciel» (ex. Sanhadja de Sraïr adar $n$ genna) et " pied $\sim$ descendant ${ }^{23}$ » (ex. Sanhadja de Sraïr iderer) se retrouvent dans plusieurs villages berbérophones appartenant aux pays des Sanhadjas de Sraïr et des Kétamas. Une variante da $n$ tayut « pied du brouillard » est attestée dans un parler rifain voisin (Igzennayen). Un second parler rifain un peu plus éloigné (Ajdir) propose la forme adad n taggut « doigt du brouillard » : il n'est pas impossible que ce composé relève d'une réinterprétation de adar " pied» en adad "doigt ». Le second élément de ces deux dernières compositions - «brouillard»- est fréquemment observé dans l'ensemble des dénominations en berbère du Maroc, et remplace « ciel » des compositions sanhadjis. Celui-ci renvoie évidemment à une observation biologique : l'hygrophylie de la salamandre. Cette distribution en bloc redondante de la composition "pied/ tombé du ciel» est très intéressante d'un point de vue dialectologique, puisqu'elle pourrait indiquer ici des phénomènes de contact anciens, voire l'origine commune de certaines populations. La Kabylie des Babors est en effet connue pour être le berceau de la dynastie berbère Koutama, connue pour peupler la région dès l'Antiquité, et célèbre pour ses faits d'armes à l'âge d'or du Califat Fatimides. La confédération Kétama du Rif marocain correspond possiblement aux descendants de guerriers Koutamas installés dans la région après la conquête de celle-ci entre le $\mathrm{x}^{\mathrm{e}}$ et le $\mathrm{XI}^{\mathrm{e}}$ siècle (Kitouni, 2013). La conservation d'un même nom berbère " pied / tombé du ciel » pour la salamandre chez des Kétamas algériens et marocain, berbérophones comme arabophones, pourrait être un indice concernant les liens historiques entre ces 
différents groupes. L'actualisation de cette composition dans le groupe des Sanhadja de Sraïr, voisins directs des Kétamas, pourrait quant à elle révéler une diffusion intra-berbère ancienne.

\subsubsection{Les motifs isolés redondants}

\section{Ce groupe est représenté par cinq éléments :}

1. Le premier type correspond à des compositions avec fiancé(e) + un second élément lié à l'eau. Celui-ci est observé en kabyle (ex. tislit wwaman « fiancée d'eau ») et en arabe jbala (ex. $\varepsilon \bar{a} r \bar{s} s$ əš-šta « fiancé de la pluie »); au masculin à partir d'éléments lexicaux de fonds arabe chez les Jbalas, contre un féminin et des mots de fonds berbère en kabyle. Les formes «amoureux du brouillard» attestées chez les Aït Waraïn dans le Moyen Atlas oriental marocain (amejnun $n$ tayut), et chez les Zkara de l'Oriental marocain (mjun $n$ tayyut) pourraient en constituer une sorte de sous-type ${ }^{24}$. En Kabylie, où ce type est représenté de manière isolée dans des parlers éloignés les uns des autres, on observe des compositions dont le second élément est « crapaud " (tislit ggemq $q^{w} e r q^{w} a r$ " fiancée du crapaud ») ou encore «entité magique invoquée les prières rogatoires pour conjurer la sécheresse ${ }^{25}$ » (tislit ggwanzar «fiancée d'Anzar»). Dans d'autres formes kabyles, on observe ce qui pourrait correspondre à des formes corrompues phonétiquement et/ou réinterprétées du nom tislit

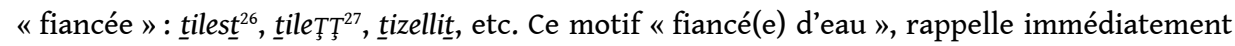
le conservatisme vasconique à l'origine du français salamandre, latin Salamandra, etc. On le retrouve également dans un parler libanais (voir sous-partie 3.2.6.), ce qui confirme son caractère circumméditerranéen, et donc appelle à la prudence afin de lui attribuer une directionalité.

2. Le deuxième motif correspond au sème de "décorée ", et s'observe en arabe du Rif central et berbère de Kabylie, partout au moyen de formes d'étymon arabe: kabyle *RQM (ex. timreqqemt), arabe kétama mzīwwqa. Ce motif pourrait être motivé par la coloration unique de l'animal. Mais une seconde motivation peut être évoquée, celle rattachant l'animal aux pratiques magiques permettant d'exceller dans l'art de la décoration de la poterie, où dans la pratique magique mobilisant une salamandre et un animal domestique en gestation afin que celui-ci donne naissance à une progéniture colorée, bariolée (voir sous-partie 4.1).

3. Le troisième motif concerne les compositions de type «transpiration d'eau de brouillard». Ce motif affiche une distribution tout à fait surprenante entre deux points éloignés : l'arabe des Jbalas de Tanger dans le Rif occidental ( $\varepsilon \bar{a} r \partial q$ əạ- $\not b a \bar{b}$ « transpiration du brouillard »), et dans un parler kabyle du sud du Djurdjura (tidit bbwwaman «transpiration d'eau»). Ces compositions sont étymologiquement réalisées au moyen d'éléments d'étymon de fonds arabe chez les Jbala, et berbère en kabyle. Il nous est impossible d'en affirmer la directionalité ni même d'affirmer que les deux formes sont liées, la forme kabyle pouvant procéder à une réinterprétation de la racine Y(DS). Une seconde forme kabyle tagudreț $p p^{w}$ aman "goutte d'eau», dont le premier élément est emprunté à l'arabe (<QṬR), est possiblement à rattacher à ce type.

4. Le quatrième type correspond à des compositions observées à travers deux exemples en kabyle et en arabe jijélien, contenant comme premier élément un type de bijoux kabyle tisemsemt gg ${ }^{w}$ aman « collier d'eau », arabe jijélien méridional qerțāt əl-jənna « boucle d'oreille du paradis ». Ces formes sont à associer au type sémantique précédent relevant du caractère singulier et jugé « beau » de la coloration et des motifs de la salamandre.

5. Le dernier type est attesté dans deux points éloignés de la Kabylie : il s'agit d'un dérivé de l'emprunt à l'arabe ȘYH «crier » correspondant au sème de "crieur, criant » (abuṣihan). Ailleurs en kabyle, cet emprunt est attesté dans la dénomination d'un autre amphibien (voir sous-partie 3.2.7). Il a pu être associé au nom de la salamandre en raison d'une confusion entre les deux animaux (voir partie 3.2.7). Les origines de ces différents parallélismes 
sémantiques en des points parfois éloignés ne nous sont pas connues, mais ceux-ci pourraient indiquer des phénomènes de contact anciens ou des convergences de substrats.

\subsubsection{Les motifs isolés non redondants}

Ceux-ci sont nombreux : ils représentent un tiers des formes recueillies. Très variés, ils nous renseignent sur la richesse des motivations sémantiques proposées par les deux continuums linguistiques et finalement sur l'hétérogénéité de la zoonymie dans les parlers arabes maghrébins et berbère-Nord:

- Arabe jijélien (a)bāyqā $\varepsilon$ ar. bu «celui à »? + «sol, terre » = "terreux ", mais aussi (a)būryūn(a) dérivé ou diminutif du nom générique du lézard.

- Arabe de l'Edough wəld ər-reād < ar. « fils du tonnerre ${ }^{28} »$.

- Berbère de l'Atlas blidéen et zénète de la Dahra « verrue, lobule » < ber. VFLḌ tasahlit afeltut «lobe », tifildi « verrue » (Haddadou, $2007: 53)$, touareg tafâdlle « excroissance de chaire » (ibid.).

- Kabyle tifiyer < ber. VFYR, majeure partie des parlers kabyles occidentaux tifiyert « chouette d'athèna ", tamazight du Moyen Atlas tifiłra "vipère ", Chenoui fiłer «serpent "; taqjunt igenni «chienne du ciel»; taqjunt uzemmur «chienne de l'oliveraie», tașabant bbwaman « récolte d'eau ${ }^{29}$ " < ar. ȘYB ; țagudreŢT ppwaman « goutte d'eau» < ar. QṬR; tabureqmant timreqqemt < ar. (bu « celui à»+) VRQM « motif » ${ }^{30}$; awlul (n igenni) < ber. VWLY « tourner»? (Haddadou, $2007: 211$ ). Nefoussa ulelli « araignée»? ou ber. VBL «forme oblongue » kabyle ablul «boudin d'argile, cheville, pénis» (Haddadou, 2007: 28), tasahlit «maïs»+ ber. «nuage, ciel »; tagrest bbwaman « hivers d'eau »; *(Y)DS > GRS < tagrest « hivers ${ }^{31} »$; tigzelt bbwaman « kyste d'eau ${ }^{32} »$.

- Tasahlit lehlal n tyerza < ar. " licite, autorisation » + < ber. « du labour » = autorisation au labour, appellation motivée par les pratiques agricoles liées à cette animal que nous décrirons dans la sous-partie 4.2.

- Chaoui des Amouchas tamaqqast $n$ waman « piquante d'eau » < ber. VQQS « piquer» Tasahlit timeqqest « scolopendre »+ « eau », rappelle les noms observés au nord de la Méditerranée mettant en avant le caractère venimeux de l'animal.

- Aït Iznassen azermița < ber. Z/ZTRM «longiforme? (kabyle aslem "poisson», azrem « serpent ", azrem « intestin ») + t ( $\left.{ }^{*} D\right)$ d'origine expressive ; un peu partout la suite ZRMD renvoi au nom du lombric (kabyle izirmed ijirmed).

- Bni Yazgha $\varepsilon \bar{a} b d$ os-sma < ar. « serviteur, homme du ciel ${ }^{33} »$.

- Sanhadji de Sraïr lmarța < ar. " glabre », axxuy n ccta < ber. " petit animal, bestiole» + < ar. «pluie».

- Kétamas berbérophones itri nt tawwet « étoile de brouillard», arabophones nəjmət əs-sma < «étoile du ciel».

- Ghomaras arabophones bərqāṭa/bərțāța d əs-sma < « tachetée du ciel ».

\subsubsection{Le contact arabe-berbère}

Le contact arabe-berbère se manifeste à travers les appellations de cet animal aussi bien en berbère qu'en arabe. Notre méconnaissance des dénominations de l'animal en arabe levantin nous empêche d'affirmer si, parmi les noms de la salamandre d'Afrique du Nord, certains pourraient être hérités des noms de sa cousine levantine Salamandra infraimmaculata ${ }^{34}$. Toutefois en berbère, plusieurs des appellations recueillies sont réalisées à partir de mots d'étymon arabe, c'est également le cas de la plupart des noms présentés pour les variétés d'arabe maghrébin concernées. Il convient donc de penser 
que le nom de la salamandre en Afrique du Nord n'est pas toujours conservateur, au point de se faire remplacer, dans de nombreux parlers, par des noms dérivés de racines arabes. C'est le cas des formes kabyles formées à partir de l'arabe RQM «motif». L'emprunt de cette racine est répandu en kabyle ${ }^{35}$; son application dans la dénomination d'un animal endémique ${ }^{36}$ est un parfait exemple de la productivité des racines empruntées à l'arabe en berbère-Nord dans les processus de création et de remplacement lexical. En berbère comme en arabe maghrébin, les dénominations de fonds arabes ou composées d'emprunts à cette langue peuvent être copiées sur d'anciens patrons sémantiques berbères (Lévy, 1996). Les motifs isolés A, C et D présentent des exemples de transferts de patrons sémantiques d'une langue à l'autre dont les directionalités restent toutefois difficiles à affirmer en l'absence du faible nombre d'occurrences de celles-ci.

Un cas intéressant de transferts de patrons sémantiques est celui des noms de la salamandre dans les Babors, formés à partir de compositions de DR «petit pied / tombée du ciel » ou « oiseau »+ « ciel ». Nous avons décrit les phénomènes d'emprunts et de ré-emprunts de formes correspondantes au sème de "petit pied / tombée du ciel » dans la sous-partie 3.2.2. Mais un second phénomène de contact doit-être signalé

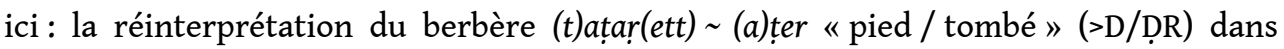
l'arabe țîr « oiseau » (>ṬYR). Cette réinterprétation est observée en berbère des Babors, dans des compositions comme țtir igenni «oiseau du ciel»; et peut-être en arabe jijélien, dans des compositions comme țirūgənnā $(n / r)$, qui paraissent composées de l'arabe tịi ${ }^{37}$ figé au berbère $\bar{u} g \partial n n \bar{a}(n)$ : țir <ber. «tombée » $\bar{u}$-<ber. préfixe vocalique marquant l'annexion d'un nom masculin singulier gənn̄a $(n)<$ ber. GNN « ciel ». Dans la forme țirūgənnār, le second élément de la composition berbère paraît contaminé par l'arabe $n \bar{a} r$ " feu ${ }^{38}$. Ces formes figées de l'arabe jijélien empruntées (/retenues) au substrat berbère sont intéressantes à plus d'un titre: elles actualisent des formes Vgənna $\sim$ Vgənnān qui ne sont pas ou plus attestées dans le berbère tasahlit voisin qui lui préfère les formes igenni «ciel " pl. igenwan. L'élément u- qui le précède semble correspondre à la voyelle de l'état d'annexion nominal du berbère qui se serait conservée ici de manière exceptionnelle ${ }^{39}$ à l'endroit où elle marquait en berbère l'annexion du nom ciel par oiseau. Dans les Babors, les variations importantes entre les différentes formes attestées au niveau de la frontière linguistique entre l'arabe et le berbère pourraient confirmer l'ancienneté de l'emprunt berbère par l'arabe, et le fait que celui-ci soit une rétention du substrat local (ancien parler berbère de la confédération) plutôt qu'un emprunt au berbère voisin.

Signalons un second phénomène observé dans quelques parlers berbères des Babors, qui est celui de la traduction de l'emprunt à l'arabe țțir « oiseau » (lui-même issue de la réinterprétation DR « pied / tombée » dans le berbère tagțit " petit oiseau, passereau ». La raison de cette traduction d'un emprunt à l'arabe en berbère illustre la grande complexité des aires de contacts intenses et anciennes ${ }^{40}$ entre l'arabe-berbère, dans lesquelles les types de bilinguisme et les situations sociolinguistiques ont varié au cours de siècles. Les tableaux suivants résument les différents phénomènes décrits ci-dessus :

\begin{tabular}{|l|l|l|l|l|}
\hline $\begin{array}{l}\text { Forme d'origine en DRR } \\
\text { «pied »+GN « ciel »: }\end{array}$ & $\rightarrow \begin{array}{l}\text { Réanalyse du ber. DQR « pied » } \\
\text { dans l'ar. TTR « oiseau » }\end{array}$
\end{tabular}$\rightarrow$\begin{tabular}{l}
$\begin{array}{l}\text { Traduction de l'ar. ṬR « oiseau » } \\
\text { dans le ber. GD « oiseau » }\end{array}$ \\
\hline
\end{tabular}




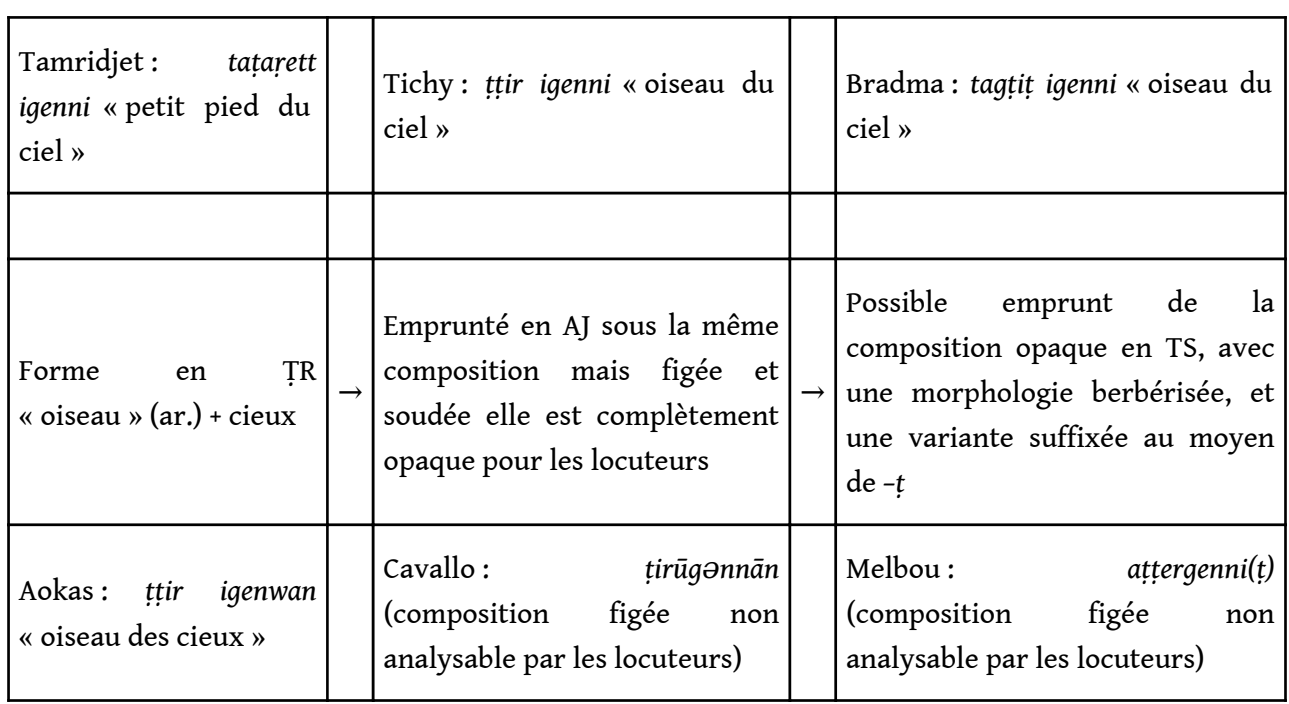

Les emprunts observés dans les noms de la salamandre en berbère du Nord doivent donc être perçus comme une conséquence du renouvellement lexical de cette langue dans un contexte de contact ancien avec l'arabe. D'un autre côté, l'arabe maghrébin propose lui aussi quelques emprunts au berbère parmi les noms qu'il donne à la salamandre, phénomène peu surprenant lorsqu'il s'agit de populations issues de l'arabisation linguistique d'anciens berbérophones. Dans les cas des dénominations de fonds arabe dans des parlers arabes maghrébins, nous ne sommes pas en mesure de déterminer dans ce travail si ceux-ci correspondent ou non à des calques de patrons issus de dénominations berbères, d'héritage de dénominations levantines, ou de créations locales. Seule l'observation des noms de la salamandre en arabe oriental nous permettra de fournir des réponses à ces interrogations.

\subsubsection{Confusions d'identifications}

40 Durant les entretiens réalisés dans le but de relever le corpus fourni dans cet article, nous avons réalisé que certaines dénominations isolées ou redondantes, semblaient provenir de confusions ou d'erreurs d'identification de l'animal avec d'autres petits vertébrés. Dans certains cas, nous avons conservé les formes données, et ce, lorsque les locuteurs ont pu nous confirmer certaines caractéristiques de l'animal (coloration, écologie). Lorsque l'identification de la salamandre par rapport à ces caractéristiques nous semblait erronée nous n'en n'avons pas tenu compte. La région qui pose le plus de difficultés semble être le Moyen Atlas marocain, dans laquelle, malgré une présence confirmée par les enquêtes des biologistes, les locuteurs interrogés ont souvent eu des difficultés à identifier la salamandre. Dans ce cas précis, nous pensons que la rareté de l'animal qui semble y présenter un mode de vie essentiellement nocturne ou troglodyte puisse expliquer la pauvreté des contacts des locuteurs avec ce dernier.

41 Ailleurs, nous avons noté, par exemple, une confusion récurrente entre la salamandre et une espèce d'ambliphème (Trogonophis wiegmanni, un amphibien apode), dans plusieurs régions situées aux frontières (occidental, oriental et méridional) de l'aire variétale kabyle. Dans ces régions, les deux animaux partagent, selon nos informateurs, la même dénomination ${ }^{41}$, et ne sont guère différenciés. C'est également le cas chez les Aït Iznassen, où le nom de la salamandre correspond à un dérivé d'une racine de fond berbère ZRM largement attestée ailleurs en berbère et largement emprunté par l'arabe 
maghrébin pour désigner de petits sauriens et autres animaux vermiformes ${ }^{42}$. Il est intéressant de mentionner le cas particulier des dénominations jijéliennes réalisées à partir de la racine BRYN d'étymon flou (ex. abūryūn). Cette racine est connue dans différents parlers berbères et arabes de la Kabylie orientale, où elle renvoie à la salamandre mais également à différentes espèces de reptiles.

Dans les parlers concernés nous avons constaté que le nom de la salamandre s'opposait à celui des reptiles au moyen de l'opposition de genre et/ou de la composition (ex. Bni

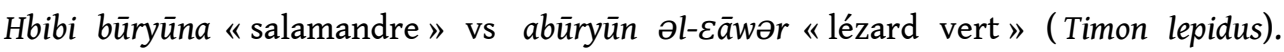
L'association des noms des lézards et de la salamandre n'est pas surprenante. Elle est attestée dans l'aire romane (Nesi, $2001: 469$ ), où le nom « lézard » renvoie soit au nom de la salamandre sans spécification (Oïl lez'ar, ibid. : 470), soit à un ensemble désignant à la fois les sauriens et les urodèles, dans lequel le nom spécifique de la salamandre est formé à partir d'une composition "lézard»+ élément spécificateur (Istro-roumain « lézard du ciel », ibid. : 472).

D'une manière assez semblable, signalons que dans le massif de l'Edough, la salamandre et l'espèce de triton endémique du massif (Pleurodeles poireti) sont toutes deux

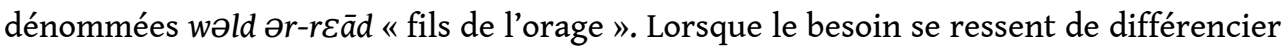
ces deux urodèles, l'adjectif qualificatif mrāngət « tacheté » est additionné au nom de la salamandre. Chez les Sanhadja de Sraïr du village de Bounjel (Zerqet), la salamandre est dénommée à partir du nom du caméléon, à travers la composition "caméléon de brouillard» (baka $n$ taggut $)$. Dans ce dernier cas, une utilisation de la salamandre semblable à celle du caméléon à travers les rituels magiques (voir sous-partie 4.2) pourrait être à l'origine de cette association.

\section{Pratiques, mythes et légendes}

La salamandre est à l'origine de nombreuses légendes et de mythes. Elle trouve également une place dans certaines pratiques agropastorales, ainsi que dans l'univers graphique des potières kabyles. La description et la compréhension de ces dictons et pratiques sont indispensables au lexicographe, puisqu'elles permettent souvent de comprendre les raisons qui ont amené à la mobilisation de tel ou tel signe sémantique dans une dénomination. Les dictons concernant la salamandre ont typiquement tendance à amplifier des caractéristiques biologiques parfois bien réelles de cet animal. C'est le cas par exemple de la légende auvergnate faisant de cet inoffensif batracien un dangereux « cracheurs » de venin mortel pour le bétail. S'il est vrai que la salamandre adulte est en mesure de secréter par sa peau des neurotoxines stéroïdes alcaloïdes ${ }^{43}$, celles-ci ne sont pas réellement dangereuses qu'en cas de consommation de l'animal. En Europe méridionale, le mythe très largement répandu d'incombustibilité de la salamandre pourrait trouver son origine dans de véritables observations biologiques: parmi celles-ci, une réelle capacité de survivre un peu plus longtemps que d'autres animaux, à l'immolation par le feu, en raison encore une fois de secrétions qu'elle produit par sa peau (Sigeaud de La Fond, 1802: 231). Nesi (2001: 468) décrit les représentations romanes du batracien ainsi: "La salamandre qui résiste au feu, venimeuse, symbole d'ambiguïté, reliée à la pluie, être magique qui vit dans les bois, peuple le fantastique, le négatif, en dépit de sa condition d'animal inoffensif et joli ». À certaines ${ }^{44}$ de ces légendes ouest-européennes dépeignant la salamandre comme une créature surnaturelle redoutée et plutôt repoussante, s'opposent les croyances nord- 
africaines que nous décrirons ci-dessous. Après quoi nous nous attarderons spécialement sur la place de cet animal dans la littérature orale à travers quelques dictons et devinettes.

Figure 6. - Figure de la salamandre à coté d'une couleuvre sur une poterie représentant les " gardiens de l'eau » (Tamridjet, Aït Bouysef, Béjäia : Algérie).

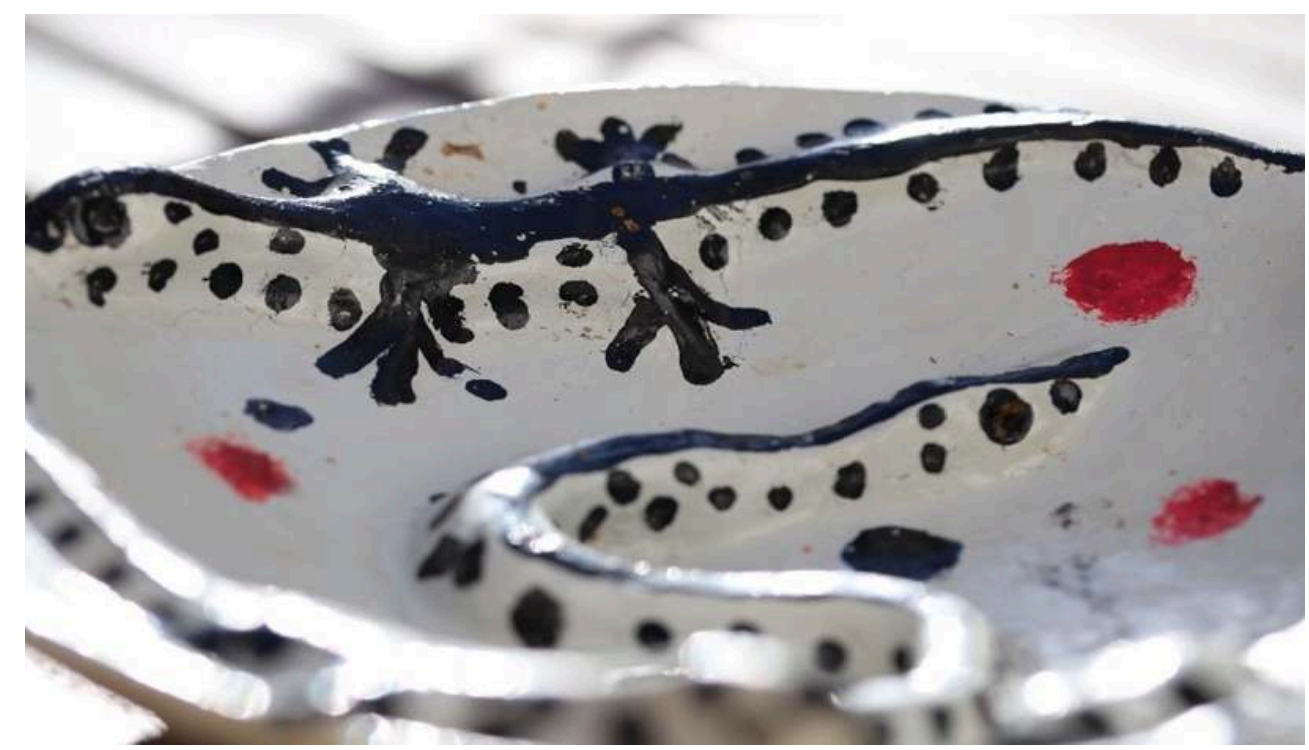

Photo : M. Garaoun.

\subsection{La salamandre dans les croyances}

Parmi les différentes aires variétales prospectées, les massifs du Djurdjura et des Babors sont ceux dans lesquels les légendes entourant la salamandre nous ont semblé être les plus riches et les plus tenaces, puisqu'encore connues et transmises aux enfants qui sont souvent convaincus de leur véracité. Nous verrons que certaines d'entre elles, plus que des croyances abstraites, pourraient être de véritables conservatismes de mythes anciens, ou encore le résultat de savoir locaux développés par ces populations rurales expertes de leurs environnements.

Les salamandres tombent du ciel par temps humide/pluvieux :

- Massif des Babors (Bni Mermi) : Yəxrəž ki yəkūn əț-ṭbāb w ən-nəẓnāz. « Elle sort par temps de brouillard ou de fine averse. »

- Massif du Djudjura (Aït Yenni) : Neqqar tetteyli-dd seg igenni mi gekkat ugeffur. « Nous disons qu'elle tombe du ciel lorsque la pluie tombe.»

- Rif central (Aït Aissi) : Idíir ma ka yəban š məng்ị f əl-wəqt dyal əš-šta, ka yəțih mən əs-sma, ka yəjib ši ḥaja, ši Eașifa wəlla. «La salamandre n'apparaît que part temps pluvieux, elle tombe $\mathrm{du}$ ciel et apporte quelque chose, une tempête où autre chose ${ }^{45}$. »

- À Freha (Djurdjura) on dit que la salamandre se cache dans les nuages avant de tomber lorsqu'il pleut. Il s'agit évidemment d'une croyance fondée sur l'apparition subite d'importantes cohortes d'animaux par temps humide.

Les salamandres sont un signe de bon ou de mauvais augure pour les cultures:

- Massif du Djurdjura (Larbaâ n At Iraten) : Tigdest $n$ waman, mi ara dd-teffey di lexrif sani ad tili ssaba dinna, ad terbeh tfellaht. "Lorsque la salamandre sort (apparait) en automne, on sait 
qu'à cet endroit on fera une bonne récolte, le travail agricole portera ses fruits. »

Tigdest $n$ waman ma yella terra aqarru-yis yer lqebla, aseggas-nni ad yili d amerbuh ad tili ssaba, ma yella terra-t anda ye yelli yițtij ur ittili ara lxir deg useggas-nni. « Lorsque la salamandre pointe sa tête vers La Mecque (vers l'ouest), l'année sera bonne, elle portera ses fruits (une bonne récolte), tandis que si elle pointe vers là où le soleil se couche (vers l'est), il n'y aura rien de bon l'année en question. "

La salamandre indique le moment à partir duquel il convient de commencer le labour en automne :

- Vallée de la Soummam (Aït Ouasif) : Ma d ifellahen, zran belli ageffur amezwaru $n$ tsemhuyt $n$ lexrif umi qqaren: tirwayin, yeserdab akal, yettara-t yeshel ityerza, ma d taydest trennu-d kan ccbaha, imi ula d nettat labud ad d-teffer yal mi ara yewwet ugeffur. "Les paysans savent reconnaître les premières pluies annonciatrices de l'automne dont ils disent : les premières pluies d'automne amollissent le sol et facilitent le labour, quant à la salamandre elle vient leur ajouter de la beauté, car elle sort obligatoirement durant ces pluies. »

- Massif du Djurdjura (Aït Zmenzer) : Ifellahen ur beddun ara tayerza deg lawan n lexrif alama teffey-d tegdest deg wakal, aya ttwalin d ttbut belli akal yerdeb yelha i tyerza. "Les paysans ne commencent pas le labour durant la saison automnal avant que la salamandre n'apparaisse (ne sorte de terre), ils voient alors que c'est le bon moment, que la terre est suffisamment tendre pour être labourée. »

- Massif des Babors: Imekrazen ttrajunt ad teffey deg akal aken ad bdun ad kerzen. "Les laboureurs attendent qu'elle (la salamandre) sorte de terre afin de commencer à labourer. » Plus qu'une croyance, cette pratique agricole est fondée sur les savoir des cultivateurs, et l'association faite entre l'apparition de cohorte de salamandre et le climat / la pluviométrie / le changement de saison.

Lécher la salamandre apporte la santé (ou la chance, le bonheur, la beauté, etc.) :

- Massif du Djurdjura (Larbaâ N At Iraten) : Win yezmer ad tt-yemceh ticki ad yescu tazmert. «Celui qui est capable de la lécher pourra peut-être obtenir la santé. »

Qqaren dið belli win imechen tagdest $n$ waman ad yes\&u zzher. « Nous disons que celui qui lèche la salamandre sera chanceux. »

Sut zik xeddment-tt, awi-d kan ad frirent ger medden s wayen icebhen. "Les anciennes le faisait (lécher la salamandre) afin de se distinguer des autres en termes de beauté. »

Il convient de penser ici que ces pratiques sont liées plus particulièrement aux différents poisons secrétés par l'animal dont la consommation par léchage que nous n'avons relevé que dans le Djudjura est partout motivée. Citons également la croyance suivante.

Lécher la salamandre confère aux potières d'amplifier le don pour le dessin :

- Massif du Djurdjura (Aït Yenni) : Mechent-tt tlawin tid ireqmen ijeqduren $n$ talext, $i$ wakken ad uxalent d izebbajin di rrqem. «Les femmes qui dessinent sur les poteries d'argile la lèchent, et ce afin de devenir douées dans le dessin. »

Tilemziyin umnent ma mechent taglimt-nni $n$ taydest icebhen ațas, ad uyalent ad lemmdent rrqem. «Les adolescentes croient que si elles lèchent la peau la peau de la très belle salamandre, elles passeront maîtres dans l'art du dessin. »

Cette opération de léchage mériterait d'être étudiée parallèlement aux conséquences des toxines secrétées par la peau de la salamandre sur la santé humaine. 
Lécher la salamandre permet de soigner les brûlures ${ }^{46}$ :

- Arrière-pays jijélien : Bəkri ǧidda kānŢ ka Ţləhs-lu kəř̌-u, əddi yəţ̧̧əhrəq b ən-nār fi yədd-u yləhs-lu yəbra. « Autrefois, grand-mère lui léchait le ventre, celui qui se brûle le bras la lèche et il guérit. »

Il est illicite de la tuer ${ }^{47}$ :

- Massif des Babors (Aït Hassaïn) : Ittuy qqaren-edd d lehram att-te zyet. «On disait autrefois qu'il était illicite de la tuer.»

Cette croyance pourrait être une survivance ancienne, attribuant à cet animal une valeur telle qu'il était interdit de lui porter atteinte.

Dans l'Atlas blidéen on pose la salamandre sur le dos d'une vache gestante dans l'espoir que celle-ci développe une robe tachetée, dans le Rif central cette pratique est appliquée sur tous les animaux domestiques.

- Kétamas: Baš məlli ka twəlləd yəkun əl-fərx mzuwwaq, zwin, bhal ider. «Afin que lorsque qu'elle mettra bas, le petit sorte décoré, beau, comme la salamandre. $»^{48}$

Dans le village de Bounjel (Zerqet, sanhadja de Sraï), où la salamandre est considérée comme un type de caméléon (appelée " caméléon de brouillard»), celle-ci fait l'objet du même genre de pratiques magiques pan-maghrébines que ce dernier dans les rituels / cérémonies de désenvoûtement. L'ensemble de ces dictons et pratiques viennent confirmer la valeur toute particulière donnée à cet animal dans les communautés rurales d'Afrique du Nord littoral. Véritable indicateur du paysan, et porte-bonheur de la potière, la salamandre joue véritablement dans ses sociétés un rôle symbolique exceptionnel incarné par des pratiques et des croyances réelles qui perdurent de nos jours.

\subsection{La salamandre dans la littérature orale}

La salamandre a également la part belle dans la littérature orale des massifs de Kabylie, où elle abonde dans les proverbes et les devinettes lesquelles mettent très souvent en valeur sa beauté ainsi que son écologie particulière. Dans les Babors, le proverbe suivant qualifie une personne experte dans son domaine :

- Treqqem tibuqualin abhhal d tațarett igenni. «Elle décore les gargoulettes comme si c'était une salamandre (comme la salamandre sait se décorer).»

Le parler des Aït Smaïl, Brahim Lxiyyer (2010) nous transmet également la devinette suivante :

- Ayyur g igenni, nettat $g$ lqaE - tataratt igenni. « La lune est dans le ciel tandis qu'elle se trouve sur le sol - la salamandre.»

Le kabyle oriental (vallée de la Soummam) Allioui (2012:187-188) nous transmet entre autres les devinettes suivantes sur cet animal :

- Ad ilin waman ad lhun yirden timzin - tidyesin. «Lorsqu'il y aura de l'eau les grains d'orge et de blé marcheront - les salamandres. »

- Yewwet ubandu ylint seg genni-tidyesin. "L'orage éclate et elles tombent du ciel- les salamandres.»

Bien d'autres aspects de la place de la salamandre dans les cultures des populations qui partagent son environnement seraient à découvrir. Parmi celles-ci, la place de la symbolique de l'animal dans la poterie et l'art ornementale kabyle (fig. 5), ainsi que les 
compréhensions anthropologiques de ces attributions. Nous avons toutefois donné ici quelques pistes concernant le rapport des populations humaines d'Afrique du Nord à cet animal. Lesquelles portent une symbolique et un regard très positif sur cet animal. Si ces données sont nécessaires pour la compréhension des motivations linguistiques de sa dénomination, elles le sont aussi pour l'ethnoécologie et l'ethnobiologie ${ }^{49}$. Nous pensons en effet que celles-ci pourraient être de bon augure pour d'éventuels projets de protection de l'animal là où celui-ci est menacé : dans la mesure où les populations pourraient être très coopératives à ce type de projets.

\section{Conclusion}

Nous avons recueilli et décrit un corpus de 100 dénominations de l'espèce Salamandra algira dans l'ensemble de ses aires de répartition algériennes et marocaines auprès de ses différentes sous-espèces décrites. Nous lui avons additionné un panorama ethnobiologique des croyances et des pratiques liées à cet animal. Cette étude nous a permis de mettre en évidence une grande diversité de dénominations en berbère comme en arabe maghrébin, pointant l'existence de dénominations attestées en bloc ou redondante sans doute de caractère ancien, parfois révélatrices d'anciens déplacements de populations ou de liens génétiques entre des aires variétales éloignées. Le croisement de ces données linguistiques et ethnobiologiques a confirmé la place symbolique importante portée par cet animal dans les croyances et les discours, gages de la stabilité, de l'ancienneté et par conséquent de l'intérêt de ses dénominations.

Nous avons relevé plusieurs phénomènes de contact linguistique, parmi lesquels des calques de compositions, des emprunts dans les deux sens (arabe > berbère comme berbère > arabe), entrainant parfois des phénomènes complexes de réinterprétations sémantiques et lexicales. Nous avons même décrit une situation où des parlers arabes maghrébins ont préservé des formes plus conservatrices que plusieurs parlers berbères voisins. Plusieurs constats faits sur ces questions de contact ne pourront cependant être complets qu'au moyen d'études des dénominations arabes levantines de la salamandre. Parmi les dénominations relevées, signalons l'intérêt particulier soulevé par l'existence d'une composition de type «fiancée de l'eau » dans des parlers berbères et arabes fortement éloignés, laquelle évoque naturellement la composition "demoiselle d'eau » pan-européenne et issue du vieux substrat vasconique, ou encore de la forme sud-libanaise "fiancée de la source». En somme, un patron de dénomination commun aux conteurs de la méditerranée, dont l'origine serait peut-être à chercher dans de vieux fonds sémantiques communs ou des contacts anciens.

BIBLIOGRAPHIE

ALLIOUI Youcef, 2012, Un grain sur le toit : énigmes et sagesses berbères de Kabylie, Paris, L'Harmattan. 
AzAïs Gabriel, 1877, Dictionnaire des idiomes romans du midi de la France, comprenant les dialectes du Haut et du Bas-Languedoc, de la Provence, etc., vol. 1, Société pour l'étude des langues romanes.

BÉRONIE Nicolas \& ANNE VIALLE Joseph, 1824, Dictionnaire du patois du Bas-Limousin, Corrèze, et plus particulièrement des environs de Tulle, Tulle, Chez L'Éditeur.

BEDRIAGA J. Von, 1883, « Beiträge zur Kenntniss der Amphibien und Reptilien der Fauna von Corsika », Archiv für Naturgeschichte, vol. 49, p. 124-273.

BeUKema Wouter, DE Pous Philip, DonAIRE David et al., 2010, « Biogeography and Contemporary Climatic Differentiation among Moroccan Salamandra algira », Biological Journal of the Linnean Society, $\mathrm{n}^{\circ} 101$, p. 626-641.

BOGAERTS Sergé, DonAIRe-BARRoso David, PASMANS Frank et al., 2013, « Do North African Fire Salamanders, Salamandra algira, Occur in Tunisia? », Herpetol, Notes, vol. 6, p. 301-306.

BоuJoT Corinne, 2001, «Corps à corps de bêtes et de gens : envenimation et représentations du corps dans le folklore français (xIX ${ }^{\mathrm{e}}-\mathrm{XX}^{\mathrm{e}}$ siècles) », Ruralia, Sciences sociales et mondes ruraux contemporains, $\mathrm{n}^{\circ} 9$.

BOUTIER Marie-Guy, 2008, « Cinq relations de base pour traiter la matière géolinguistique : réflexions à partir de l'expérience de l'Atlas linguistique de la Wallonie », Estudis Romànics, vol. 30, p. 301-310.

CHAKER Salem, 1996, « Emphase », Encyclopédie berbère, vol. 17.

Corriente Federico, Pereira Christophe \& Vicente Ángeles (éds), (2017), Dictionnaire du faisceau dialectal arabe andalou : perspectives phraséologiques et étymologiques, Walter de Gruyter.

DALlET Jean-Marie, 1982, Dictionnaire kabyl-français, parler des At Mangellat Algérie, Paris, Société d'études linguistiques et anthropologiques de France.

EL ARIFI Samir, 2016, Tamazight de l'Atlas blidéen, Alger, Haut-Commissariat à l'Amazighité.

EsCoRIzA Daniel, 2007, «Els amfibis de còrsego i sardenya », Treballs de la Societat Catalana d'Herpetologia, $\mathrm{n}^{\circ} 4$.

ESCORIZA Daniel \& BEN HASSINE Jihene, 2014, « Salamandra algira (North African Fire Salamander ", The Herpetological Bulletin, $\mathrm{n}^{\circ}$ 128, p. 24-25.

HERNANDEZ Axel \& EsCoRIZA Daniel, 2019, « A New Subspecies of African Fire Salamander Salamandra algira (Urodela, Salamandridae) from the Middle Atlas Mountains, Morocco », ZooKeys, vol. 893, p. 143.

HICKEY Raymond \& PUPPEL Stanislav, 2010, Language History and Linguistic Modelling: A Festschrift for Jacek Fisiak on His 60th Birthday, Berlin, Walter de Gruyter.

JôNAIN Pierre Abraham, 1869, Dictionnaire du patois saintongeais, Paris, Chez L'auteur.

Kossmann Maarten, 1996, « Du nouveau à propos du nom de l'aiguille », Études et documents berbères, vol. 14, p. 97-105.

KIRECHE Ouerdya, 2010, Étude comparative du vocabulaire fondamental entre les parlers de Sidi Ali Bounab et Souk El Tenine. Analyse morphologique et sémantique, dissertation doctorale, Tizi-Ouzou, Université de Tizi-Ouzou-Mouloud Mammeri.

Kitouni Hosni, 2013, La Kabylie orientale dans l'histoire. Pays des Kutuma et guerre coloniale, Paris, L'Harmattan. 
LÉvY Simon, 1996, « Repères pour une histoire linguistique du Maroc », Estudios de dialectología norteafricana y andalusí, vol. 1, p. 127-137.

LXIYYER Brahim, 2010, Yal azamul is\&a azal, Iriten $n$ YimaziYen, Anamek $n$ umawal $n$ rrqem $n$ temnadt $n$ Ayt Smacel, Tidukkla Tadelsant Adrar n Fad.

MAMOUNI Fayza \& MEBAREK Tasacdit, 2015, Tasledt takennayt $n$ uktawal yerzan ismawen $n$ yi ibeecac d yifrax di tmurt $n$ leqbayel, Mémoire de master, Université de Tizi-Ouzou-Mouloud Mammeri.

MAUDUYT Lubin, 1825, Le " vocabulaire poitevin », Paris, Édition critique d'après Poitiers.

NAÏT-ZERRAD Kamal, 1999, Dictionnaire des racines berbères (formes attestées), vol. II - C-DEN, Paris / Louvain, Peeters Publishers.

NAÏT-ZERRAD Kamal, 2002, Dictionnaire des racines berbères (formes attestées), vol. III - D-GEY, Centre de recherche berbère, INALCO, Paris / Louvain, Peeters Publishers.

NESI Annalisa, 2001, «Les désignations romanes de la salamandre », dans Atlas linguistique roman, vol. 2.a : Commentaire et carte, Roma, Istituto Poligrafico e Zecca dello Stato.

ORPUSTAN Jean-Baptiste, 2006, « Noms basques des plantes, champignons et animaux d'Irati Iratiko landare, onddo eta ihizien izenak euskaraz (Essai de lexicographie basque) », Bulletin du Musée basque, Société des Amis du Musée basque.

Rossi Mario, 2006, Dictionnaire étymologique et ethnologique des parlers brionnais : Bourgogne du Sud, Publibook.

SIGAUD DE LA FOND Joseph-Aignan, 1802, Dictionnaire des merveilles de la nature, vol. 3, Paris, Chez Delaplace.

TARBÉ Prosper, 1851, Recherches sur l'histoire du langage et des patois de Champagne, vol. 1., Imprimerie de P. Régnier.

TERMITE Marinella, 2019, «Écrire avec les animaux : les choix inattendus de Barbey d'Aurevilly », Studia Universitatis Babes-Bolyai-Philologia, vol. LXIV, $\mathrm{n}^{\circ}$ 3, p. 41-54.

VENY Joan, 2019, Petit atles lingüístic del domini català, vol. 7, Barcelone, Institut d'Estudis Catalans.

\section{NOTES}

1. Je souhaite vivement remercier Evgeniya Gutova, Sofiane Smaïl et les relecteurs anonymes pour leurs commentaires sur une version précédente de ce travail.

2. Ce comportement ne se vérifie pas chez toutes les espèces: Salamandra lanzai (espèce alpine) par exemple, a développé une reproduction vivipare à la suite de laquelle la progéniture est mis au monde sous une forme identique à celle des adultes ce qui ne l'oblige pas à passer par un stade aquatique.

3. Il y a cinq à six millions d'années avant notre ère.

4. En dehors de son aire de répartition, l'animal, lorsqu'il est connu, est généralement dénommé au moyen de son nom générique en arabe classique : smāndāl.

5. Thèse financée par l'École pratique des hautes études (EPHE) et le Laboratoire de langues, langages et cultures d'Afrique (LLACAN).

6. On pourra difficilement reprocher aux auteurs ces erreurs, tant l'identification de l'animal peut être malaisée, notamment chez des populations parfois restées rurales mais dont les savoirs sur la faune qui les entourent ont diminués en raison d'un mode de vie centré sur des valeurs 
urbaines. Nous aborderons l'épineux problème des confusions d'identification dans la partie 3.2.7.

7. Les dénominations senhadjis nous ont aimablement été données par Evgeniya Gutova, que nous remercions vivement.

8. La variation dans les dénominations de cet animal est si importante qu'il nous est impossible d'affirmer avoir systématiquement récolter l'ensemble des formes en usage dans les différents points d'enquête. Nous nous excusons par avance pour les formes erronées ou occultées par ce corpus.

9. Sofiane Smaill, communication personnelle

10. Kania Rabdi, communication personnelle.

11. Samir Tighzert, communication personnelle.

12. Durant le séminaire de linguistique berbère dirigé par Amina Mettouchi (lundi 17 mai 2021).

13. La semi-vocalisation de [f] est un phénomène attesté en berbère (voir Kossmann, 1996).

14. Signalons également taferdeddist "graisse de conserve» (fer-+ DS ?) chez les Ntifa (Naït-Zerrad, 2002: 616). La préfixation d'une radicale $f-$, pourrait également correspondre en berbère à un préfixe expressif (Naït-Zerrad, 1999 ; 2002), ce qui viendrait expliquer le sémantisme péjoratif du mot chleuh. La préfixation à des fins expressives de la racine DS est par ailleurs attestée au moyen de l'élément $\varepsilon$ - dans aceddis « gros ventre » (kabyle), « ventre » (tamazight du Moyen Atlas).

15. Voir kabyle occidental tafunast «vache »vs Timezrit (kabyle extrême-occidental) tafunazt (Kireche, 2010 : 95).

16. Forme non intégrée dans notre corpus en raison du refus du locuteur de nous donner sa confédération d'origine.

17. L'arabe dit jijélien.

18. Dans l'aire romane, les dénominations de la salamandre liées à la pluie sont courantes dans la région alpine (Nesi, 2001). On retrouve également mais plus rarement des noms associant l'animal au ciel en roman oriental (ibid.).

19. La réalisation assourdie de la dentale emphatique $/ \mathrm{d} /(>/ \mathrm{t} /)$ correspond à une caractéristique phonétique bien connue des parlers arabes et berbères de la Kabylie orientale.

20. Signalons également la proximité de la composition utilisée dans le parler tasahlit des Aït Ouaret Ouali pour désigner l'arc-en-ciel : ațar igenni (litt. « jambe du ciel ») dans laquelle il apparaît clairement que l'idée de «jambe » est associée à un certain mouvement, une "chute, descente » du ciel.

21. Ce qui est indiqué ici par le degré important de jonction des deux éléments de la composition (perte des voyelles initiales), ainsi que par leur conservation en arabe jijélien (qui tire ces formes de son substrat berbère).

22. Le nom aylal a disparu de ce parler en synchronie.

23. Kétamas (Ayt Aissi) idir ider . Cette forme connait dans ce parler une vocalisation différente de celle du mot pied : adar. C'est également le cas de certaines formes en arabe et en berbère des Babors : țergenni (Aït Laâlam), țīrūgennan (Bni Mermi), etc. Il y aurait peut-être lieu de rattacher ces formes des Babors au nom d'action du verbe berbère «descendre » (tasahlit : ațray), voir à une forme conjuguée de ce verbe figé (tasahlit ițer « il est descendu») : ce qui indiquerait que le processus de réinterprétation sémantique de la racine désignant la salamandre de l'action de « chuter, descendre » vers celui de " pied » pourrait s'être produit parallèlement dans les Babors et le Rif central, avec une conservation d'une potentielle forme d'origine "descendre, fait de descendre " dans les parlers les plus conservateurs du berbère des Babors (tasahlit-est), dans l'arabe local qui l'a anciennement emprunté et dans un unique parler berbère d'une fraction kétama pour le Rif central.

24. Ces formes sont réalisées à partir de compositions hybrides entre un emprunt à l'arabe pour le premier élément « amoureux » et un de fond berbère pour «brouillard ». Le premier élément 
de la composition récoltée chez les Zkara - mjun - n'est pas directement identifiable comme un dérivé de l'arabe məjnūn "possédé, envouté, fou (d'amour)». Nous l'avons associé à celui-ci en raison de la forme du parler des Aït Waraïn amejnun, identifié par les locuteurs à cet étymon emprunté, auquel le sens donné est " amoureux ».

25. Anzar est un terme pan-berbère renvoyant à "pluie». En kabyle, celui-ci a pris le sens particulier de l'entité mythique dont on implore la grâce dans les prières réalisées pour faire tomber la pluie. Cette cérémonie, et le mythe qui lui est associé, varient d'un village à un autre, mais l'on y retrouve souvent le personnage de la fiancée d'Anzar, une jeune femme dont la main est symboliquement offerte par la communauté à cette entité afin de mettre fin à la sécheresse. Il est possible que la salamandre entre ou entrait dans ce rituel chez les At Amran.

26. Réinterprétation de SLY « fiancé » en LS « langue »?

27. Corruption de LS en LT par évolution du [s] en [ts] au contact du [ $\theta]$ final.

28. Cette dénomination n'est pas sans rappeler les formes « fils de la poule » pour « œuf» (arabe Algérois et bougiote) et «fils de la mer » pour « poisson » (arabe tunisien). Ces compositions ont sans doute pour origine le tabou linguistique qui entourent ces entités, largement utilisé dans les pratiques magiques maghrébines. Dans les différents parlers concernés par les deux compositions précédemment citées, celles-ci s'emploient à chaque fois à côté d'un nom non taboué : Béjaïa $\varepsilon \bar{a} \underline{d} m a$ « œuf », vieil algérois bìṭa, Tunis hūta " poisson » : ce n'est pas le cas dans le massif de l'Edough où la composition en « fils de + nom » a été relevée seule (à moins que le tabou n'ai contraint notre informateur à ne pas nous donner l'équivalent non tabouisé). Remarquons que la salamandre atteste de dénominations tabouisées également dans plusieurs langues romanes du nord de la péninsule italique (Nesi, 2001) : dans ces langues toutefois, le nom tabouisé est souvent formé à partir d'un nom de parenté (ibid.).

29. Forme clairement motivée sur les croyances/savoirs associant l'observation de la salamandre en automne à une bonne année agraire (voir sous-partie 4.1).

30. Ailleurs en kabyle la même racine empruntée est fréquemment à l'origine du nom du chardonneret élégant (Carduelis carduelis).

31. La motivation de cette dénomination se trouve dans le cycle biologique de l'animal, qui apparaît aux yeux des hommes surtout durant les épisodes pluvieux de la saison hivernale (voir sous-partie 2.2).

32. GẒL < tigzelt « kyste, rein, rognon ».

33. Cette dénomination tout à fait isolée est l'une des plus intéressantes de notre corpus, dans la mesure où celle-ci relaie une véritable anthropomorphisation de l'animal. Elle renforce le caractère si particulier de l'interprétation nord-africaine de la salamandre comme une créature magique céleste.

34. Nous avons recueilli deux dénominations glanées auprès d'informateurs libanais : Aïn Aanous (parler druze) ḥasān buhardun, et dans le sud du Liban Earūs él-Eayn « fiancée de la source » (nous ne disposons pas ici de localité précise, mais présentons cette forme pour son intérêt indéniable pour notre propos). Dans les parlers bédouins de Syrie, nous n'avons trouvé que des rétentions de l'arabe classique. Il est tout à fait possible que la salamandre dispose au Levant d'un nombre de dénominations tout à fait comparable à celui de l'Afrique du Nord et donc d'un grand intérêt.

35. En raison de son nombre relativement important de dérivés nominaux et verbaux : kabyle occidental (At Mangellet, Dallet, 1982: 732) rrqem «dessiner, colorier», urqim «dessiné », aberreqmuc « bariolé », etc.

36. En berbère tasahlit, cette racine de fond arabe sert à désigner différents passereaux bariolés : chez les Aït Bouysef de la fraction des Aït Laârch, tabureqmant désigne la sous-espèce de mésange bleue endémique à l'Afrique septentrionale (Parus caeruleus ultramarinus), tandis que dans la fraction des Aït Djemaâ, agțị tabureqmayt renvoie au chardonneret (Carduelis carduelis). Ce qui vient témoigner du remplacement lexical par des emprunts à l'arabe même dans un lexique que l'on aurait pu supposer conservateur (espèces animales sauvages endémiques). 
37. Nous ne pensons pas que tiir- dans ce composé puisse être attribué à l'arabe ṬYR «planer, voler, oiseau » : en raison de son figement total dans une composition hybride dont l'emprunt au substrat berbère est peut-être ancien et surtout en connaissance de la dénomination idir «salamandre", cognat recueilli dans un parler sanhadja de Sraïr (Maroc). Toutefois, certains locuteurs réanalysent cet élément berbère figé comme provenant de l'arabe « oiseau ».

38. D'après la légende locale selon laquelle l'animal soigne les brûlures (voir sous-partie 4.1).

39. Nous n'avons pas connaissance d'autres exemples de sa conservation dans des emprunts au berbère de l'arabe maghrébin.

40. $>1200$ ans dans la Kabylie des Babors.

41. A $\underline{\text { bușih }}$ ab bușihan < ar bu qualificateur + ȘYH « crier » = « criant, crieur ».

42. Dans la Kabylie des Babors, celle-ci renvoie uniformément au lombric: Tasahlit des Aït Mhend izirmeț, arabe jijélien (Bni Mâad) azərməț, etc. La racine ZRM renvoie partout à de petits sauriens et est empruntée par l'arabe à l'échelle pan-maghrébine (ex. arabe marocain zərmūmìya, arabe tunisien zərzūmìya « lézard»).

43. Le samandarin, le samandaridin et le samanderon.

44. Tous les dits et dénominations romanes de la salamandre ne font pas cependant écho à une représentation négative de cet animal. Signalons par exemple le nom composé "garde fontaines " récolté dans les Pyrénées (Nesi, 2001: 472), qui «attribue à l'animal la tâche de divinité tutélaire des sources (ibid.). L'existence de ces quelques dénominations positives de la salamandre dans les compositions vasconiques pourrait indiquer la facture récente de ces formes négatives.

45. Evgeniya Gutova, communication personnelle.

46. Cette association entre la salamandre et le feu rappelle les mythes d'Europe occidentale.

47. Cet interdit doit être considéré comme d'ordre spirituel dans la mesure où le terme employé est lehram « interdit religieux ».

48. Evgeniya Gutova, communication personnelle.

49. Lors d'une présentation de ce travail au séminaire du LACNAD, le 4 novembre 2020, Daniela Merolla a également souligné l'importance de ces «représentations nord-africaines » de la salamandre du point de vue de l'éthnocritique animale et de la poétique écologiste. Il y aurait là beaucoup d'idées à développer sur les terrains des écologistes et anthropologues.

\section{RÉSUMÉS}

Salamandra algira est la seule espèce de salamandre africaine. Ce batracien ne se rencontre que dans quelques massifs montagneux d'Algérie et du Maroc. Nous avons recueilli cent dénominations de la salamandre parmi les variétés de berbère et d'arabe pratiquées dans ces montagnes. Puis, nous avons décrit ce corpus en regardant ses motivations sémantiques, avant de l'interroger sous le prisme du contact linguistique arabe-berbère; avec pour problématique principale la détermination des stratégies de dénominations de cet animal endémique à l'Afrique du Nord par les populations arabophones. Cette enquête linguistique est suivie d'une courte présentation ethnobiologique des dictons et pratiques autours de la salamandre d'Afrique du Nord.

Salamandra algira is the only African salamander species. This amphibian can only be found in a few mountain ranges of Algeria and Morocco. We collected one hundred salamander 
denominations among the Berber and Arabic varieties practice in these moutains. Then, we described this corpus by looking at its semantic motivations, before questionning it under the prism of Arab-Berber linguistic contact; with the main problematic of determining the naming strategies of this North African endemic animal by the Arabic-speaking populations. This linguistic investigation is followed by a short ethnobiological presentation of the myths and the practices turning around the North African salamander.

\section{INDEX}

Keywords : North African fire Salamander, Salamandra algira, Berber, Arabic, contact, lexicography, Algeria, Morocco

Mots-clés : salamandre d'Algérie, Salamandra algira, berbère, arabe, contact, lexicographie, Algérie, Maroc

\section{AUTEUR}

\section{MASSINISSA GARAOUN}

Doctorant à l'École pratique des hautes études, Laboratoire des langues et langages et cultures d'Afrique

massinissagaraoun@gmail.com 